\title{
On the orthogonal subgrid scale pressure stabilization of finite deformation $\mathrm{J} 2$ plasticity
}

\author{
C. Agelet de Saracibar ${ }^{\mathrm{a}, *}$, M. Chiumenti ${ }^{\mathrm{a}}$, Q. Valverde ${ }^{\mathrm{b}}$, M. Cervera ${ }^{\text {a }}$ \\ a International Center for Numerical Methods in Engineering (CIMNE), Universidad Politécnica de Cataluña (UPC), \\ Edificio C1, Campus Norte, Gran Capitán s/n, E-08034 Barcelona, Spain \\ b Departamento de Ingenieria, Sección Ingenieria Mecánica, Pontificia Universidad Católica del Perú, Lima, Perú, Chile
}

Accepted 25 April 2005

\begin{abstract}
The use of stabilization methods is becoming an increasingly well-accepted technique due to their success in dealing with numerous numerical pathologies that arise in a variety of applications in computational mechanics.

In this paper a multiscale finite element method technique to deal with pressure stabilization of nearly incompressibility problems in nonlinear solid mechanics at finite deformations is presented. A J2-flow theory plasticity model at finite deformations is considered. A mixed formulation involving pressure and displacement fields is used as starting point. Within the finite element discretization setting, continuous linear interpolation for both fields is considered. To overcome the Babuška-Brezzi stability condition, a multiscale stabilization method based on the orthogonal subgrid scale (OSGS) technique is introduced. A suitable nonlinear expression of the stabilization parameter is proposed. The main advantage of the method is the possibility of using linear triangular or tetrahedral finite elements, which are easy to generate and, therefore, very convenient for practical industrial applications.

Numerical results obtained using the OSGS stabilization technique are compared with results provided by the P1 standard Galerkin displacements linear triangular/tetrahedral element, P1/P1 standard mixed linear displacements/linear pressure triangular/tetrahedral element and Q1/P0 mixed bilinear/trilinear displacements/constant pressure quadrilateral/hexahedral element for 2D/3D nearly incompressible problems in the context of a nonlinear finite deformation $\mathrm{J} 2$ plasticity model.
\end{abstract}

(C) 2005 Elsevier B.V. All rights reserved.

Keywords: Multiscale methods; Subgrid scale methods; Orthogonal subgrid scale methods; Stabilized finite element methods; Stabilization; Incompressibility; Plasticity; Finite deformation

\footnotetext{
* Corresponding author. Tel.: +34934016495; fax: +34934016517.

E-mail addresses: agelet@cimne.upc.es (C. Agelet de Saracibar), chiument@cimne.upc.es (M. Chiumenti), qvalver@pucp.edu.pe (Q. Valverde), cervera@cimne.upc.es (M. Cervera).
} 


\section{Introduction}

The use of stabilized methods is becoming an increasingly well-accepted technique due to their success in dealing with numerous numerical pathologies that arise in a variety of applications in computational mechanics. This paper deals with the application of multiscale methods, in particular the orthogonal subgrid scale (OSGS) method, to the pressure stabilization of nearly incompressibility problems in nonlinear solid mechanics at finite deformations using low order finite elements. A Finite deformation $\mathbf{J} 2$ plasticity model is considered. The goal is to consistently derive, within the framework of the OSGS method, a modified variational mixed formulation of the original problem with enhanced stability properties.

It is well known that the standard irreductible Galerkin finite element method with low-order piecewise polynomials perform miserably in nearly incompressible problems, exhibiting spurious wild oscillations of the mean pressure and leading to a response which is almost completely locked due to the incompressibility constraint. In the computational literature these devastating numerical difficulties are referred to as locking phenomena. Actually, the exact incompressibility problem does not admit an irreductible formulation and, consequently, a mixed displacement/pressure framework is necessary in that case. Even though, many standard mixed finite element formulations, particularly those using low order interpolations, also perform poorly or totally fail to perform for nearly incompressibility or incompressibility problems, producing results throughly polluted by spurious oscillations of the pressure.

To overcome these difficulties, over the years different strategies were suggested to reduce or avoid volumetric locking and pressure oscillations in finite element solutions. For an engineering oriented presenta-
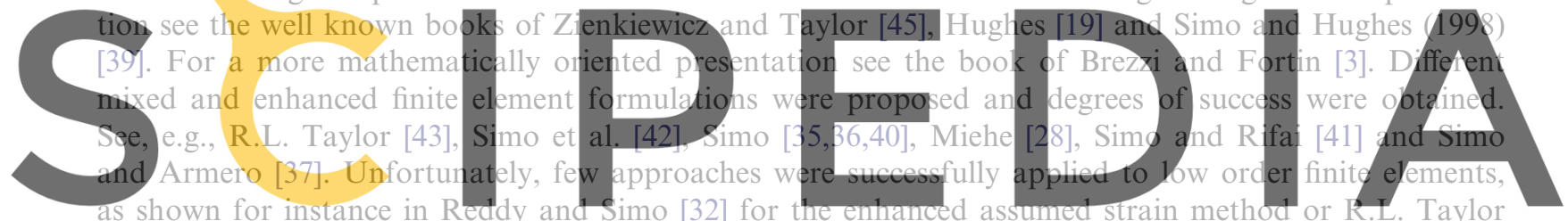

[43] for the mixed method. This was due to the strictness of the inf-sup or Ladyzhenskaya-Babuška-Brezzi

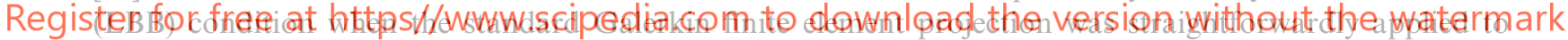

mixed low order finite elements, as it imposes severe restrictions on the compatibility of the interpolations used for the displacement and pressure fields $[3,4.5]$. One significant effort in that direction was the so called mini element [1], an attractive linear displacement/pressure triangle enhanced with a cubic displacement bubble function. The mini-element satisfies the LBB condition, but it is only marginally stable and it does not perform very well in many practical situations. Despite these not very good satisfactory results, there still exists a great practical interest in the use of stable low order elements, mainly motivated by the fact that, nowadays, tetrahedral finite element meshes are relatively easy to generate for real life complex geometries. Therefore, stabilization techniques for low order finite elements is a very active research area in solid mechanics. Some recent formulations have been proposed by Zienkiewicz et al. [46], Klaas et al. [24], Oñate et al. [29,30], Maniatty et al. [26,27], Maniatty and Liu [25], Reese and Wriggers [33], Reese et al. [34], and Ramesh and Maniatty [31]. In Zienkiewicz et al. [46] a stabilization term, arising from a fractional step method, is introduced using a mixed displacement/pressure formulation and linear triangles and tetrahedra, within the framework of explicit dynamic codes for solids. In Klaas et al. [24] a stabilized formulation for large deformation elasticity using P1/P1 elements was presented, where stabilization was attained by adding a mesh-dependent stabilization term, which can be viewed as a perturbation of the test functions, leading to a Galerkin least square (GLS) stabilized discrete weak form. This formulation has been recently extended to elastoplastic finite deformation problems by Ramesh and Maniatty [31]. An extension to higher order interpolation functions, using a local reconstruction method to construct part of the stabilization terms, was presented by Maniatty et al. [27]. Maniatty et al. [26] also presented a stabilized formulation for steady state flow problems to simulate forming problems such as drawing. In Oñate 
et al. $[29,30]$ a stabilization term was derived using a finite calculus (FIC) formulation and applied for linear static and explicit and semi-implicit dynamic finite element analysis of bulk and impact problems using linear triangles and tetrahedra.

On the other hand, research on stabilization methods for incompressibility, as well as other phenomena, in computational fluid dynamics (CFD) has been always in the front line of research because of the innumerable practical applications of the field [2,4,15,21,22]. In Hughes [20] and Hughes et al. [23] the variational multiscale method was introduced as a new computational mechanics paradigma to address stabilization problems in CFD. Within the multiscale method it is assumed that there is a component of the continuous (exact) solution which can not be captured by the finite element solution. This component which is not captured by the finite element solution is called the subgrid scale or the subscale. The consideration of this subgrid scale leads to a modified variational formulation with enhanced stability properties and allows the use of a convenient mixed velocity/pressure equal linear interpolation. Since their inception, multiscale methods have been extensively and successfully used in CFD. In Codina [13,14] the Orthogonal Subgrid Scales (OSGS) method was introduced, leading to better sustained and better performing stabilization procedures.

In computational solid mechanics (CSM), variational multiscale techniques have been used by Garikipati and Hughes $[16,17]$ in strain localization problems. Recently, a variational multiscale stabilization method based on the OSGS has been applied to both incompressibility and nearly incompressibility problems in small deformations elasticity by Valverde et al. [44], Chiumenti et al. [11] and Christ et al. [10], J2 plasticity by Valverde et al. [44], Chiumenti et al. [12], Cervera et al. [6] and Christ et al. [10], softening and localization in J2 plasticity model by Cervera et al. [9

The goal of this paper

OSGS method, to incomp ter

formation $\mathrm{J} 2$ plasticity

The outline of the rematning of
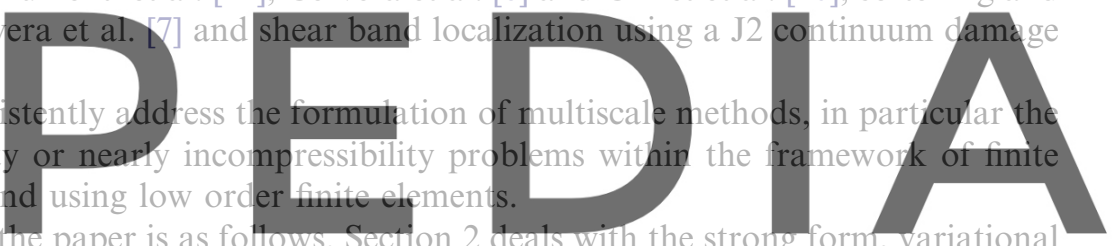

form and discrete variational form of the mixed formulation of the nearly incompressibility nonlinear prob-

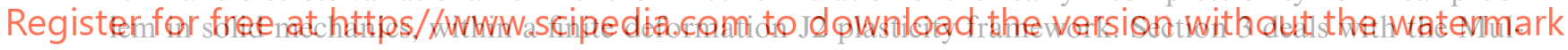

tiscale formulation of $\mathrm{J} 2$ plasticity models at finite deformations. Within the multiscale technique framework, the variational multiscale forms are derived, a nonlinear expression for the stabilization parameter is proposed and the subsgrid scale displacements are approximated using an OSGS stabilization method. Some computational and implementation aspects are discussed in Section 4. An assessment of the behaviour of the formulation is presented in Section 5, where some representative numerical simulations are shown and compared with results obtained using P1 Galerkin displacements linear triangular (2D) or tetrahedral (3D) elements, P1/P1 mixed linear displacement/linear pressure triangular (2D) or tetrahedral (3D) elements and Q1/P0 bilinear (2D) or trilinear (3D) displacement/constant pressure quadrilateral (2D) or hexahedral (3D) elements. Some concluding remarks are addressed in Section 6. Finally, after an acknowledgement section, Appendix A including the derivation of the linearization of the variational momentum balance residual has been included.

\section{Nearly incompressibility problem in finite deformation $\mathbf{J 2}$ plasticity}

Let us begin introducing some standard notation. Let $\Omega$ be an open and bounded domain of $\mathbb{R}^{n_{\text {dim }}}$, where $n_{\mathrm{dim}}$ is the number of space dimensions, $\bar{\Omega}$ its closure and $\Gamma$ its boundary which is considered split into two disjoint sets such that $\Gamma=\overline{\partial \Omega_{u} \cup \partial \Omega_{t}}$ and $\partial \Omega_{u} \cap \partial \Omega_{t}=\emptyset$. The space of square integrable functions in $\Omega$ is denoted by $L^{2}(\Omega)$ and the space of functions of which its derivatives up to order $m \geqslant 0$ (integer) belong to $L^{2}(\Omega)$ by $H^{m}(\Omega)$. The space $H_{0}^{m}(\Omega)$ consists of those functions that belong to $H^{m}(\Omega)$ and vanish on 
$\partial \Omega_{u}$. Bold characters are used to denote vector counterpart of all these spaces. The $L^{2}$ inner product in $\Omega$ and in $\partial \Omega$ are denoted by $(\cdot, \cdot)$ and $(\cdot,)_{\partial \Omega}$, respectively. Hereafter, orthogonality will be understood with respect to this product.

\subsection{Strong form}

Let us consider an elastoplastic (isotropic) material model at finite deformations within the framework of phenomenological models derived from a micromechanical description of single-crystal metal plasticity. An essential feature of this micromechanical description is the introduction of an intermediate local stress-free configuration, relative to which the elastic response of the material is characterized. From a phenomenological standpoint this notion leads to a local multiplicative decomposition of the deformation gradient of the form $\mathbf{F}=\mathbf{F}^{\mathrm{e}} \mathbf{F}^{\mathrm{p}}$, where $\mathbf{F}^{\mathrm{e}}$ and $\mathbf{F}^{\mathrm{p}}$ denote the elastic and plastic deformation gradients, respectively. In addition, in accordance with a standard assumption in metal plasticity, we assume that the plastic flow is isochoric and therefore the following relations hold: $\operatorname{det}\left[\mathbb{F}^{\mathrm{p}}\right]=1, J:=\operatorname{det}[\mathbb{F}]=\operatorname{det}\left[\mathbb{F}^{\mathrm{e}}\right]$. Consistent with the assumptions of isotropy, isochoric plastic flow and the notion of an intermediate stress-free configuration, we characterize the stress response by an uncoupled volumetric/isochoric stored-energy function of the form [39]

$$
W\left(J, \overline{\mathbf{b}}^{\mathrm{e}}\right)=U(J)+\bar{W}\left(\overline{\mathbf{b}}^{\mathrm{e}}\right)
$$

where the volumetric part $U(J)$ of the stored energy function $W\left(J, \overline{\mathbf{b}}^{\mathrm{e}}\right)$ is a convex function of the determi-

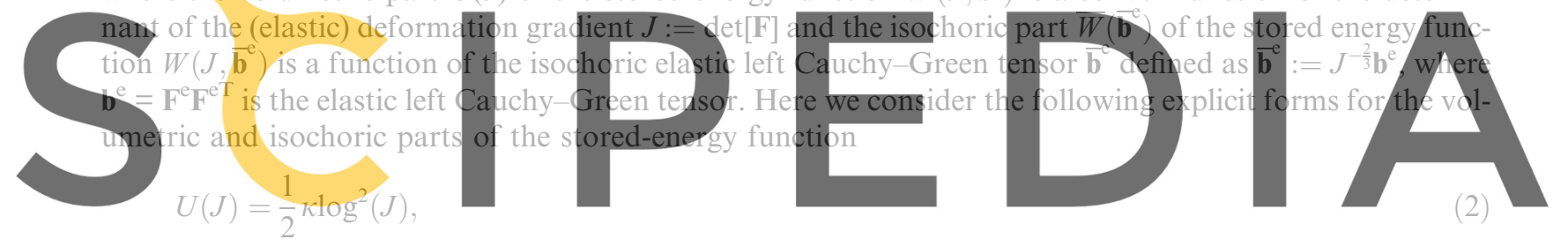

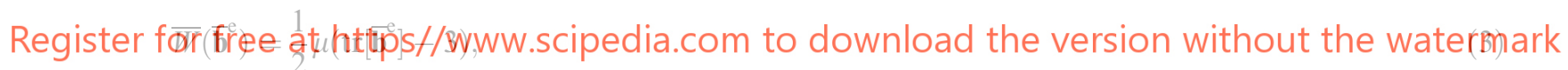

where $\mu>0$ and $\kappa>0$ are interpreted as the shear modulus and the bulk modulus, and $\operatorname{tr}[\cdot]=1:[\cdot]$ denotes the spatial trace operator, where $\mathbf{1}$ is the second order unit tensor.

Following a standard derivation $[39,42]$ the (mixed) Kirchhoff stress tensor can be written as

$$
\tau(\mathbf{u}, \pi)=\pi \mathbf{1}+\mathbf{s}(\mathbf{u}),
$$

where the Kirchhoff pressure $\pi:=\frac{1}{3} \operatorname{tr}[\tau(\mathbf{u}, \pi)]$, to be viewed as an independent variable, and the deviatoric component of the (mixed) Kirchhoff stress tensor $\mathbf{s}(\mathbf{u}):=\operatorname{dev}[\tau(\mathbf{u}, \pi)]$ take the form

$$
\begin{aligned}
& \pi=J U^{\prime}(J), \\
& \mathbf{s}(\mathbf{u})=\mu \operatorname{dev}\left[\overline{\mathbf{b}}^{\mathrm{e}}\right] .
\end{aligned}
$$

Note that the uncoupled volumetric/isochoric stored-energy function results in uncoupled volumetric/ deviatoric stress response, where $\operatorname{dev}[\cdot]:=\left(\mathbf{I}-\frac{1}{3} \mathbf{1} \otimes \mathbf{1}\right):[\cdot]$ is the spatial deviatoric operator and $\mathbf{I}$ is the fourth-order identity tensor. $U^{\prime}(J)$ denotes the derivative of $U(J)$ with respect to $J$. In what follows, it will be implicitly assumed that $J=J(\mathbf{u})$ is a function of the displacement field $\mathbf{u}$.

Appropriate boundary conditions will be taken as $\mathbf{u}=\overline{\mathbf{u}}$ on $\partial \Omega_{u}$ and $\tau \mathbf{F}^{-\mathrm{T}} \mathbf{N}=\overline{\mathbf{t}}^{N}$ on $\partial \Omega_{t}$, where $\overline{\mathbf{u}}: \partial \Omega_{u} \rightarrow \mathbb{R}^{n_{\mathrm{dim}}}$ and $\overline{\mathbf{t}}^{N}: \partial \Omega_{t} \rightarrow \mathbb{R}^{n_{\mathrm{dim}}}$ are the prescribed displacement and nominal traction vectors, respectively, and $\mathbf{N}$ is the unit outer normal field to $\partial \Omega$. Consider the infinite-dimensional spaces $\mathscr{V}=\{\mathbf{u} \in$ $\mathbf{H}^{1}(\Omega) \mid \mathbf{u}=\overline{\mathbf{u}}$ on $\left.\partial \Omega_{u}\right\}$ and $\mathscr{Q}=L^{2}(\Omega)$ for the displacement and Kirchhoff pressure fields, respectively. We shall be interested also in the space $\mathscr{W}=\mathscr{V} \times \mathscr{Q}$. Then the strong form of the mixed formulation for the 
nearly incompressibility problem in finite deformation solid mechanics consists in finding a displacement field $\mathbf{u} \in \mathscr{V}$ and a Kirchhoff pressure field $\pi \in \mathscr{Q}$ such that

$$
\begin{aligned}
& J \nabla\left(J^{-1} \pi\right)+J \nabla \cdot\left(J^{-1} \mathbf{s}(\mathbf{u})\right)+\mathbf{f}=\mathbf{0} \text { in } \Omega, \\
& \pi-J U^{\prime}(J)=0 \text { in } \Omega,
\end{aligned}
$$

where $\mathbf{f}: \Omega \rightarrow \mathbb{R}^{n_{\mathrm{dim}}}$ is the prescribed body force per unit reference volume vector, $\nabla(\cdot)$ denotes the spatial gradient operator and $\nabla \cdot(\cdot)$ denotes the spatial divergence operator.

Using an abstract compact notation, the problem defined by (4) and (5) can be written as: find $\mathbf{U} \in \mathscr{W}$ such that

$$
\mathscr{L}(\mathbf{U})=\mathscr{F} \text { in } \Omega,
$$

where $\mathbf{U}, \mathscr{L}(\mathbf{U})$ and $\mathscr{F}$ are defined as

$$
\mathbf{U}=\left[\begin{array}{l}
\mathbf{u} \\
\pi
\end{array}\right], \quad \mathscr{L}(\mathbf{U})=\left[\begin{array}{c}
-J \nabla\left(J^{-1} \pi\right)-J \nabla \cdot\left(J^{-1} \mathbf{S}(\mathbf{u})\right) \\
-\pi+J U^{\prime}(J)
\end{array}\right], \quad \mathscr{F}=\left[\begin{array}{l}
\mathbf{f} \\
0
\end{array}\right] .
$$

\subsection{Variational form}

Consider the infinite-dimensional space $\mathscr{V}_{0}=\mathbf{H}_{0}^{1}(\Omega)$. We shall be interested also in the space $\mathscr{W}_{0}=\mathscr{V}_{0} \times 2$. Then the variational formulation of the nearly incompressibility problem in solid mechanics
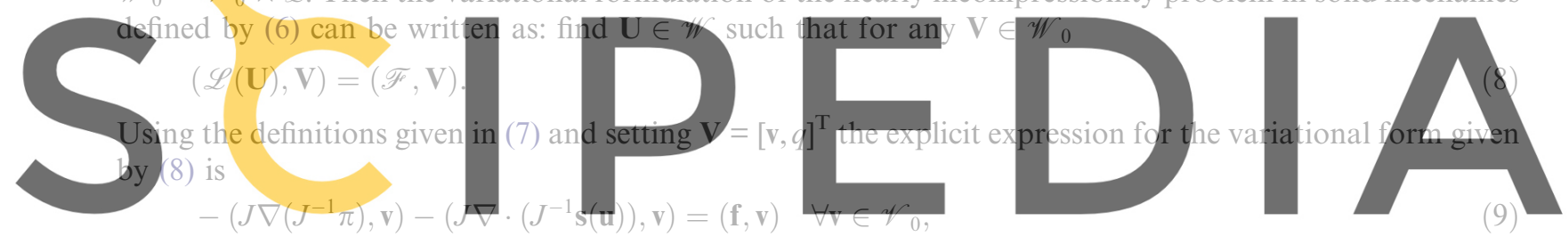

Register for free at https//Www.scipedia.com to download the version without the watermark

Integrating by parts the left-hand side of (9), the above variational forms can be written as:

$$
\begin{aligned}
& (\pi, \nabla \cdot \mathbf{v})+\left(\mathbf{s}(\mathbf{u}), \nabla^{s} \mathbf{v}\right)=l(\mathbf{v}) \quad \forall \mathbf{v} \in \mathscr{V}_{0}, \\
& -(\pi, q)+\left(J U^{\prime}(J), q\right)=0 \forall q \in \mathscr{Q},
\end{aligned}
$$

where the operator $l(\mathbf{v}):=(\mathbf{f}, \mathbf{v})+\left(\overline{\mathbf{t}}^{N}, \mathbf{v}\right)_{\partial \Omega}$ has been introduced and the fact that $\mathbf{s}(\mathbf{u})$ is a symmetric tensor has been used.

Introducing an abstract compact notation, the variational formulation of the nearly incompressibility problem in solid mechanics given by (8) can be alternatively written as: find $\mathbf{U} \in \mathscr{W}$ such that for any $\mathbf{V} \in \mathscr{W}_{0}$

$$
B(\mathbf{U}, \mathbf{V})=L(\mathbf{V}),
$$

where

$$
\begin{aligned}
& B(\mathbf{U}, \mathbf{V})=(\pi, \nabla \cdot \mathbf{v})+\left(\mathbf{s}(\mathbf{u}), \nabla^{s} \mathbf{v}\right)-(\pi, q)+\left(J U^{\prime}(J), q\right), \\
& L(\mathbf{V})=l(\mathbf{v}) .
\end{aligned}
$$

\subsection{Discrete variational form}

The standard Galerkin projection of this variational problem is now straightforward. Let $\mathscr{P}_{h}$ denote a finite element partition of the domain $\Omega$. The diameter of an element domain $e \in \mathscr{P}_{h}$ is denoted by $h_{e}$ 
and the diameter of the finite element partition by $h=\max \left\{h_{e} \mid e \in \mathscr{P}_{h}\right\}$. We can now construct conforming finite element spaces $\mathscr{V}_{h} \subset \mathscr{V}, \mathscr{Q}_{h} \subset \mathscr{Q}$ and $\mathscr{W}_{h}=\mathscr{V}_{h} \times \mathscr{Q}_{h}$ in the usual manner, as well as the corresponding subspaces $\mathscr{V}_{h, 0} \subset \mathscr{V}_{0}$ and $\mathscr{W}_{h, 0}=\mathscr{V}_{h, 0} \times \mathscr{Q}_{h}$. In principle, functions in $\mathscr{V}_{h}$ are continuous, whereas functions in $\mathscr{Q}_{h}$ not necessarily. Likewise, the polynomial orders of these spaces may be different. Then, the discrete version of the variational problem (11) consists in finding $\mathbf{U}_{h} \in \mathscr{W}_{h}$ such that for any $\mathbf{V}_{h} \in \mathscr{W}_{h, 0}$

$$
B\left(\mathbf{U}_{h}, \mathbf{V}_{h}\right)=L\left(\mathbf{V}_{h}\right) .
$$

Remark 1. As it is well known, convenient displacement-pressure interpolations, such as equal linear interpolations, turn out to violate the inf-sup or Babuška-Brezzi condition. To circumvent this condition, the idea now is to replace the discrete variational problem (14) by a suitable discrete stabilized variational problem, such that the variational form $B$ is replaced by a possibly mesh dependent variational form $B_{\text {stab }}$ with enhanced stability properties. Eventually, the linear form $L$ may be also replaced by a possibly mesh dependent form $L_{\text {stab. }}$. This is done in the next sections through the introduction of the subgrid scale method.

\section{Multiscale formulation of $\mathbf{J} 2$ plasticity models at finite deformations}

In this section, a stabilization of the mixed formulation of J2 plasticity models at finite deformations is
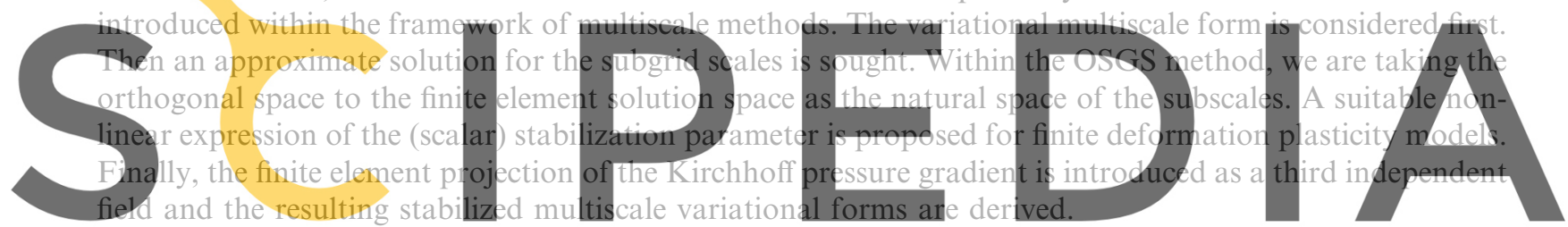

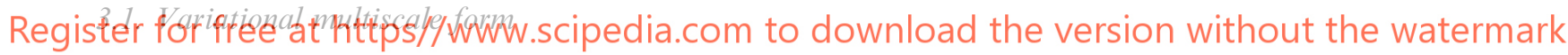

\subsubsection{Multiscale approach}

Within the paradigmatic framework of the multiscale methods introduced by Hughes [20], the subgrid scale method seeks to approximate the effect of the component of the continuous solution which can not be captured by the finite element mesh on the discrete finite element solution. The unresolved component is referred to as the subgrid scale or subscale. Let $\mathscr{W}=\mathscr{W}_{h} \oplus \widetilde{\mathscr{W}}$, where $\widetilde{\mathscr{W}}$ is any suitable space to complete $\mathscr{W}_{h}$ in $\mathscr{W}$. Obviously, $\widetilde{W}$ is an infinite-dimensional space, but once the final method will be formulated, it is approximated by a finite-dimensional space, although we will keep the same symbol for it in order to simplify the notation. We will refer to $\widetilde{\mathscr{W}}$ as the space of the subgrid scales or the space of the subscales. Likewise, let $\mathscr{W}_{0}=\mathscr{W}_{h, 0} \oplus \widetilde{\mathscr{W}}_{0}$, with $\widetilde{\mathscr{W}}_{0}$ any space to complete $\mathscr{W}_{h, 0}$ in $\mathscr{W}_{0}$. With the above definitions in hand, we consider that there is a component $\widetilde{\mathbf{U}} \in \widetilde{\mathscr{W}}$ of the exact continuous solution $\mathbf{U} \in \mathscr{W}$ which cannot be captured by the solution provided by the finite element method $\mathbf{U}_{h} \in \mathscr{W}_{h}$, such that

$$
\mathbf{U}=\mathbf{U}_{h}+\widetilde{\mathbf{U}},
$$

where for the nearly incompressibility problem in solid mechanics $\mathbf{U}, \mathbf{U}_{h}$ and $\widetilde{\mathbf{U}}$ take the form

$$
\mathbf{U}=\left[\begin{array}{l}
\mathbf{u} \\
\pi
\end{array}\right], \quad \mathbf{U}_{h}=\left[\begin{array}{c}
\mathbf{u}_{h} \\
\pi_{h}
\end{array}\right], \quad \widetilde{\mathbf{U}}=\left[\begin{array}{c}
\widetilde{\mathbf{u}} \\
\tilde{\pi}
\end{array}\right],
$$

where $\mathbf{U}_{h}$ is the resolved component of the primary variable provided by the finite element solution and may be interpreted as the projection of the exact solution $\mathbf{U}$ onto the finite-dimensional space introduced by the 
finite element discretization. Therefore $\widetilde{\mathbf{U}}$ is the component of the exact continuous solution which can not be captured by the discrete finite element solution. It is now necessary to introduce some additional finitedimensional subspaces associated to the previously defined infinite-dimensional spaces.

\subsubsection{Variational multiscale approach}

Introducing the split of $\mathbf{U}$ given by (15), the variational multiscale formulation of the nearly incompressibility problem in solid mechanics given by (11) can be written as: find $\mathbf{U}_{h} \in \mathscr{W}_{h}$ and $\widetilde{\mathbf{U}} \in \widetilde{\mathscr{W}}$ such that

$$
\begin{aligned}
& B\left(\mathbf{U}_{h}+\widetilde{\mathbf{U}}, \mathbf{V}_{h}\right)=L\left(\mathbf{V}_{h}\right) \quad \forall \mathbf{V}_{h} \in \mathscr{W}_{h, 0}, \\
& B\left(\mathbf{U}_{h}+\widetilde{\mathbf{U}}, \widetilde{\mathbf{V}}\right)=L(\widetilde{\mathbf{V}}) \quad \forall \widetilde{\mathbf{V}} \in \widetilde{\mathscr{W}}_{0} .
\end{aligned}
$$

Assuming that $\tilde{\pi}=0$, i.e. that the exact Kirchhoff pressure field may be captured by the finite element solution and therefore the subgrid scale associated to the Kirchhoff pressure is zero, the variational multiscale formulation given by (17) and (18) can be written as: find $\left(\mathbf{u}_{h}, \pi_{h}\right) \in \mathscr{W}_{h}$ and $(\widetilde{\mathbf{u}}, 0) \in \widetilde{W}$ such that

$$
\begin{aligned}
& \left(\pi_{h}, \nabla \cdot \mathbf{y}_{h}\right)+\left(\mathbf{s}\left(\mathbf{u}_{h}+\widetilde{\mathbf{u}}\right), \nabla^{s} \mathbf{v}_{h}\right)=l\left(\mathbf{v}_{h}\right) \quad \forall \mathbf{v}_{h} \in \mathscr{V}_{h, 0}, \\
& \left(J\left(\mathbf{u}_{h}+\widetilde{\mathbf{u}}\right) U^{\prime}\left(J\left(\mathbf{u}_{h}+\widetilde{\mathbf{u}}\right)\right)-\pi_{h}, q_{h}\right)=0 \quad \forall q_{h} \in \mathscr{V}_{h}, \\
& \left(\pi_{h}, \nabla \cdot \widetilde{\mathbf{v}}\right)+\left(\mathbf{s}\left(\mathbf{u}_{h}+\widetilde{\mathbf{u}}\right), \nabla^{s} \widetilde{\mathbf{v}}\right)=l(\widetilde{\mathbf{v}}) \quad \forall \widetilde{\mathbf{v}} \in \widetilde{\mathscr{V}}_{0} .
\end{aligned}
$$

Note that due to the fact that the subscale associated to the Kirchhoff pressure field has been assumed to be

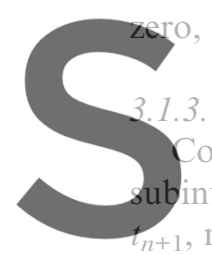

the second equation
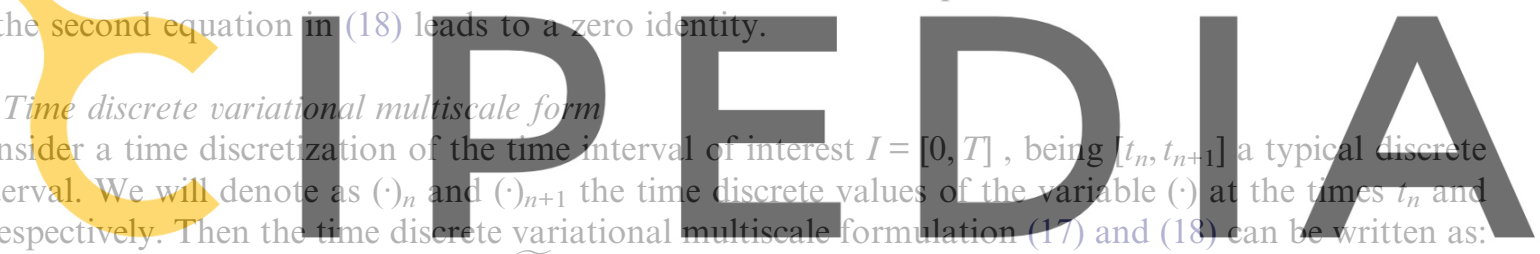

find $\left(\mathbf{u}_{h, n+1}, \pi_{h, n+1}\right) \in \mathscr{W}_{h}$ and $\left(\widetilde{\mathbf{u}}_{n+1}, 0\right) \in \mathscr{W}$ such that:

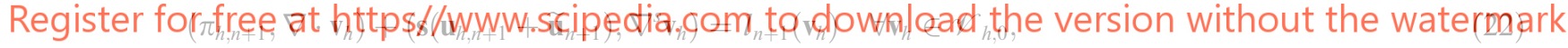

$$
\begin{aligned}
& \left(J\left(\mathbf{u}_{h, n+1}+\widetilde{\mathbf{u}}_{h+1}\right) U^{\prime}\left(J\left(\mathbf{u}_{h, n+1}+\widetilde{\mathbf{u}}_{n+1}\right)\right)-\pi_{h, n+1}, q_{h}\right)=0 \quad \forall q_{h} \in \mathscr{Q}_{h}, \\
& \left(\pi_{h, n+1}, \nabla \cdot \widetilde{\mathbf{v}}\right)+\left(\mathbf{s}\left(\mathbf{u}_{h, n+1}+\widetilde{\mathbf{u}}_{n+1}\right), \nabla^{s} \widetilde{\mathbf{v}}\right)=l_{n+1}(\widetilde{\mathbf{v}}) \quad \forall \widetilde{\mathbf{v}} \in \widetilde{\mathscr{V}},
\end{aligned}
$$

where $l_{n+1}\left(\mathbf{v}_{h}\right):=\left(\mathbf{f}, \mathbf{v}_{h}\right)+\left(\overline{\mathbf{t}}_{n+1}^{N}, \mathbf{v}_{h}\right)_{\partial \Omega}$ and $l_{n+1}(\widetilde{\mathbf{v}}):=(\mathbf{f}, \widetilde{\mathbf{v}})+\left(\overline{\mathbf{t}}_{n+1}^{N}, \widetilde{\mathbf{v}}\right)_{\partial \Omega}$. Note that the last equation represents an infinite-dimensional variational form for the subgrid scales.

Proposition 2 (Deviatoric stress and Kirchhoff pressure split). Consider the linearization of $J\left(\mathbf{u}_{h, n+1}+\widetilde{\mathbf{u}}_{n+1}\right) U^{\prime}\left(J\left(\mathbf{u}_{h, n+1}+\widetilde{\mathbf{u}}_{n+1}\right)\right)$ and $\mathbf{s}\left(\mathbf{u}_{h, n+1}+\widetilde{\mathbf{u}}_{n+1}\right)$. To deal with this linearizations we will perform a Taylor series expansion about the current displacement solution provided by the finite element approximation and keep only the linear terms in the subgrid scales.

Linearization of $\left.\left[J U^{\prime}(J)\right]\right|_{\mathbf{u}_{h, n+1+\tilde{u}_{n+1}}}$. The linearization of the Kirchhoff pressure $\left.\left[J U^{\prime}(J)\right]\right|_{\mathbf{u}_{h, n+1+\tilde{u}_{n+1}}}$ yields

$$
\left.\left[J U^{\prime}(J)\right]\right|_{\mathbf{u}_{h, n+1+\tilde{u}_{n+1}}}=\left.\left[J U^{\prime}(J)\right]\right|_{\mathbf{u}_{h, n+1}}+\left.D\left[J U^{\prime}(J)\right]\right|_{\mathbf{u}_{h, n+1}} \cdot \widetilde{\mathbf{u}}_{n+1},
$$

where the directional derivative $\left.D\left[J U^{\prime}(J)\right]\right|_{\mathbf{u}_{h, n+1}} \cdot \widetilde{\mathbf{u}}_{n+1}$ along the direction $\widetilde{\mathbf{u}}_{n+1}$ evaluated at $\mathbf{u}_{h, n+1}$ takes the form

$$
\left.D\left[J U^{\prime}(J)\right]\right|_{\mathbf{u}_{h, n+1}} \cdot \widetilde{\mathbf{u}}_{n+1}=\left.\left.\left[J U^{\prime}(J)\right]^{\prime}\right|_{\mathbf{u}_{h, n+1}} D J\right|_{\mathbf{u}_{h, n+1}} \cdot \tilde{\mathbf{u}}_{n+1}
$$

and, taking into account that the directional derivative $\left.D J\right|_{\mathbf{u}_{h, n+1}} \cdot \widetilde{\mathbf{u}}_{n+1}$ takes the form

$$
\left.D J\right|_{\mathbf{u}_{h, n+1}} \cdot \widetilde{\mathbf{u}}_{n+1}=J\left(\mathbf{u}_{h, n+1}\right) \nabla \cdot \widetilde{\mathbf{u}}_{n+1},
$$


where $\nabla \cdot(\cdot)$ denotes the spatial divergence operator, the linearization of the (exact) volumetric term $\left.\left[J U^{\prime}(J)\right]\right|_{\mathbf{u}_{h, n+1+\tilde{\mathbf{u}}_{n+1}}}$ yields

$$
\left.\left[J U^{\prime}(J)\right]\right|_{\mathbf{u}_{h, n+1+\tilde{u}_{n+1}}}=\left.\left[J U^{\prime}(J)\right]\right|_{\mathbf{u}_{h, n+1}}+\left.\left.\left[J U^{\prime}(J)\right]^{\prime}\right|_{\mathbf{u}_{h, n+1}} J\right|_{\mathbf{u}_{h, n+1}} \nabla \cdot \widetilde{\mathbf{u}}_{n+1} .
$$

For the explicit form of the volumetric part of the stored-energy function given by (2), the following relations hold $J U^{\prime}(J)=\kappa \log (J),\left[J U^{\prime}(J)\right]^{\prime}=\kappa J^{-1}$ and, introducing the short notation $J_{n+1}:=\left.J\right|_{\mathbf{u}_{h, n+1+\tilde{u}_{n+1}}}$ and $J_{h, n+1}:=\left.J\right|_{\mathbf{u}_{h, n+1}}$, the linearization yields

$$
\log J_{n+1}=\log J_{h, n+1}+\nabla \cdot \widetilde{\mathbf{u}}_{n+1}
$$

and, therefore, the following relation holds $J_{n+1}=J_{h, n+1} \exp \left(\nabla \cdot \widetilde{\mathbf{u}}_{n+1}\right)$.

Linearization of $\mathbf{s}\left(\mathbf{u}_{h, n+1}+\widetilde{\mathbf{u}}_{n+1}\right)$. The linearization of the deviatoric stress $\mathbf{s}\left(\mathbf{u}_{h, n+1}+\widetilde{\mathbf{u}}_{n+1}\right)$ yields

$$
\mathbf{s}\left(\mathbf{u}_{h, n+1}+\widetilde{\mathbf{u}}_{n+1}\right)=\mathbf{s}\left(\mathbf{u}_{h, n+1}\right)+D \mathbf{s}\left(\mathbf{u}_{h, n+1}\right) \cdot \nabla^{s} \widetilde{\mathbf{u}}_{n+1}
$$

where

$$
D \mathbf{s}\left(\mathbf{u}_{h, n+1}\right) \cdot \nabla^{s} \widetilde{\mathbf{u}}_{n+1}=\mathbf{c}_{h, n+1}^{\mathrm{dev}}: \nabla^{s} \widetilde{\mathbf{u}}_{n+1},
$$

where, assuming plastic loading, $\mathbf{c}_{h, n+1}^{\mathrm{dev}}$ denotes the deviatoric part of the consistent (algorithmic) tangent moduli, e.g., for plastic loading the deviatoric part of the consistent elastoplastic moduli arising from the lineari-

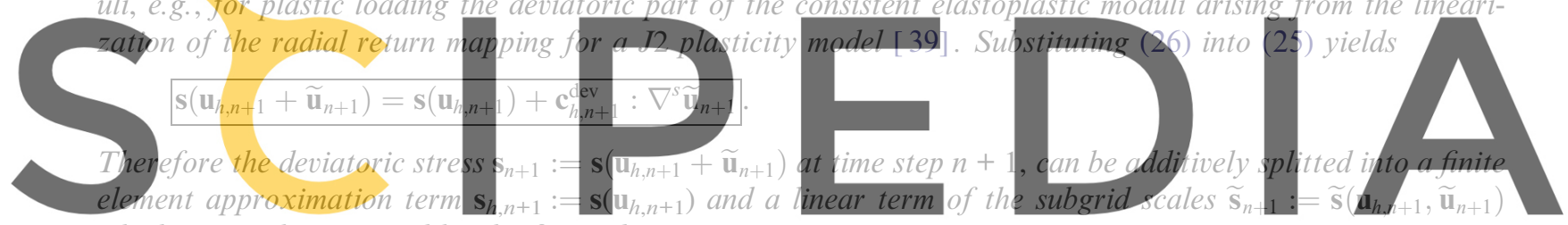

which cannot be captured by the finite element approximation

\section{Register for.fre $\boldsymbol{B}_{h}$ at 1 httips//./www.scipedia.com to download the version without the watermark}

where

$$
\begin{aligned}
& \mathbf{s}_{n+1}=\mathbf{s}\left(\mathbf{u}_{h, n+1}+\widetilde{\mathbf{u}}_{n+1}\right), \\
& \mathbf{s}_{h, n+1}=\mathbf{s}\left(\mathbf{u}_{h, n+1}\right)=\mu \operatorname{dev}\left[\overline{\mathbf{b}}_{h, n+1}^{\mathrm{e}}\right], \\
& \widetilde{\mathbf{s}}_{n+1}=\mathbf{c}_{h, n+1}^{\mathrm{dev}}: \nabla^{s} \widetilde{\mathbf{u}}_{n+1} .
\end{aligned}
$$

Remark 3. The deviatoric stress tensor associated to the subgrid scales $\widetilde{\mathbf{s}}_{n+1}:=\widetilde{\mathbf{s}}\left(\mathbf{u}_{h, n+1}, \widetilde{\mathbf{u}}_{n+1}\right)$, which in general will be a function of the current displacements provided by the finite element solution, should be viewed as an incremental perturbation relative to the current stress tensor solution provided by the finite element approximation.

Using the additive split of the deviatoric stress tensor $\mathbf{s}_{n+1}$, substituting (27) into the time discrete variational forms (22), (23) (divided by the bulk modulus) and (24), the time discrete variational multiscale formulation (17) and (18) can be written as: find $\left(\mathbf{u}_{h, n+1}, \pi_{h, n+1}\right) \in \mathscr{W}_{h}$ and $\left(\widetilde{\mathbf{u}}_{n+1}, 0\right) \in \widetilde{\mathscr{W}}$ such that

$$
\begin{aligned}
& \left(\pi_{h, n+1}, \nabla \cdot \mathbf{v}_{h}\right)+\left(\mathbf{s}_{h, n+1}, \nabla^{s} \mathbf{v}_{h}\right)+\left(\widetilde{\mathbf{s}}_{n+1}, \nabla^{s} \mathbf{v}_{h}\right)=l_{n+1}\left(\mathbf{v}_{h}\right) \quad \forall \mathbf{v}_{h} \in \mathscr{V}_{h, 0}, \\
& \left(\log J_{h, n+1}-\frac{1}{\kappa} \pi_{h, n+1}, q_{h}\right)+\left(\nabla \cdot \widetilde{\mathbf{u}}_{n+1}, q_{h}\right)=0 \quad \forall q_{h} \in \mathscr{Q}_{h}, \\
& \left(\pi_{h, n+1}, \nabla \cdot \widetilde{\mathbf{v}}\right)+\left(\mathbf{s}_{h, n+1}, \nabla^{s} \widetilde{\mathbf{v}}\right)+\left(\widetilde{\mathbf{s}}_{n+1}, \nabla^{s} \widetilde{\mathbf{v}}\right)=l_{n+1}(\widetilde{\mathbf{v}}) \quad \forall \widetilde{\mathbf{v}} \in \widetilde{\mathscr{V}}_{0} .
\end{aligned}
$$


The goals now are twofold. First, to find an approximate solution for the (displacement) subgrid scales within the infinite-dimensional variational problem (33). For this, the infinite-dimensional space of the subgrid scales will be approximated by a finite-dimensional space which, within the OSGS method, will be the orthogonal space to the finite element space. Second, to substitute the approximate solution for the subgrid scales into the finite-dimensional variational problem given by (31) and (32).

\subsection{Orthogonal subgrid scales (OSGS)}

\subsubsection{Algorithmic variational form for the subgrid scales}

Integrating by parts within each element the first two terms of the left-hand side of (33) and taking into account the equilibrium of (exact) tractions at the interelement boundaries yields

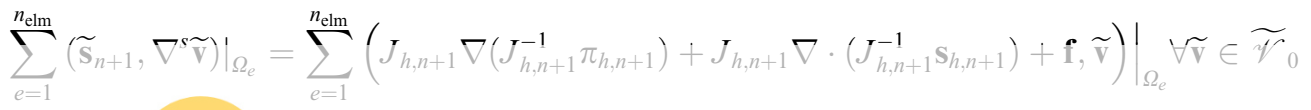

which at the element level yields $\forall \widetilde{\mathbf{v}} \in \widetilde{\mathscr{V}} 0$

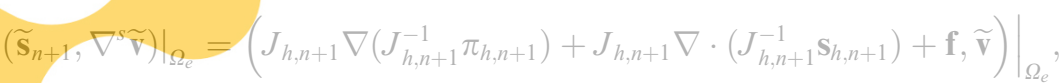

where the right-hand side term represents the variational form of the residual of the momentum balance equation given by the finite element approximation.
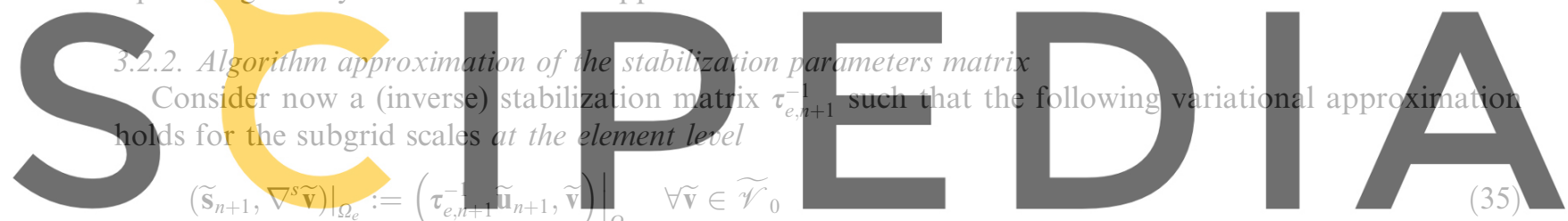

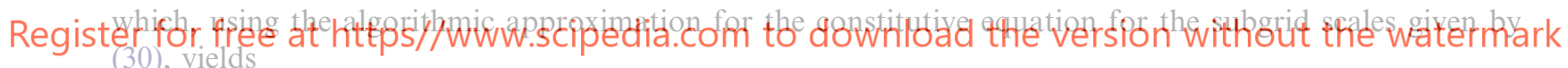

$$
\left.\left(\mathbf{c}_{h, n+1}^{\mathrm{dev}}: \nabla^{s} \widetilde{\mathbf{u}}_{n+1}, \nabla^{s} \widetilde{\mathbf{v}}\right)\right|_{\Omega_{e}}:=\left.\left(\tau_{e, n+1}^{-1} \widetilde{\mathbf{u}}_{n+1}, \widetilde{\mathbf{v}}\right)\right|_{\Omega_{e}} \quad \nabla \widetilde{v} \in \widetilde{V}_{0} .
$$

Consider now a J2-plastic flow model with isotropic hardening. A summary of the J2-plastic flow model with isotropic hardening is shown in Table 1. Table 2 shows the main steps involved in the radial return mapping algorithm and Table 3 shows the expression of the consistent tangent elastoplastic moduli considering a linear isotropic hardening law [35-37,39,40,42].

Proposition 4 (Algorithmic approximation of the scalar stabilization parameter). In order to introduce an approximate solution for the subgrid scales $\widetilde{\mathbf{u}}_{n+1}$, the following simple secant approximation to the constitutive equation for the subgrid scales is considered

$$
\widetilde{\mathbf{s}}_{n+1}:=\mathbf{c}_{h, n+1}^{\mathrm{dev}}: \nabla^{s} \widetilde{\mathbf{u}}_{n+1} \approx 2 \widetilde{\mu}_{n+1} \operatorname{dev}\left[\nabla^{s} \widetilde{\mathbf{u}}_{n+1}\right],
$$

where, assuming plastic loading, the deviatoric part of the consistent elastoplastic moduli has been replaced by $\mathbf{c}_{h, n+1}^{\mathrm{dev}} \approx 2 \widetilde{\mu}_{n+1}\left(\square-\frac{1}{3} \mathbf{1} \otimes \mathbf{1}\right)$ and the (secant) subgrid scale shear modulus for plastic loading is defined as

$$
\widetilde{\mu}_{n+1}:=\mu J_{h, n+1}^{-2 / 3} \frac{\left\|\operatorname{dev}\left[\overline{\mathbf{b}}_{h, n+1}^{\mathrm{e}}\right]\right\|}{\left\|\operatorname{dev}\left[\overline{\mathbf{b}}_{h, n+1}\right]\right\|} .
$$

Substituting (37) into (36) yields the following variational approximation for the subscales at the element level 
Table 1

Hyperelastic J2-flow model at finite deformations

(i) Multiplicative decomposition of the deformation gradient $\mathbf{F}=\mathbf{F}^{\mathrm{e}} \mathbf{F}^{\mathrm{p}}$

(ii) Free energy with linear isotropic hardening

$$
\begin{aligned}
& \psi\left(J, \overline{\mathbf{b}}^{\mathrm{e}}, \xi\right):=U(J)+\bar{W}\left(\overline{\mathbf{b}}^{\mathrm{e}}\right)+\mathscr{H}(\xi) \\
& U(J)=\frac{1}{2} \kappa \log ^{2}(J), J=\operatorname{det}[\mathbf{F}]=\operatorname{det}\left[\mathbf{F}^{\mathrm{e}}\right] \\
& \bar{W}\left(\overline{\mathbf{b}}^{\mathrm{e}}\right)=\frac{1}{2} \mu\left(\operatorname{tr}\left[\overline{\mathbf{b}}^{\mathrm{e}}\right]-3\right), \overline{\mathbf{b}}^{\mathrm{e}}=J^{-\frac{2}{3}} \mathbf{b}^{\mathrm{e}}, \mathbf{b}^{\mathrm{e}}=\mathbf{F}^{\mathrm{e}} \mathbf{F}^{\mathrm{eT}} \\
& \mathscr{H}(\xi)=\frac{1}{2} H \xi^{2}-\left(\sigma_{0}-\sigma_{\infty}\right)(\xi-(1-\exp (-\delta \xi)) / \delta)
\end{aligned}
$$

(iii) Mixed hyperelastic and hardening response

$$
\begin{aligned}
& \tau(\mathbf{u}, \pi)=\pi \mathbf{1}+\mathbf{s}(\mathbf{u}), \pi=J U^{\prime}(J), \mathbf{s}(\mathbf{u})=\mu \operatorname{dev}\left[\overline{\mathbf{b}}^{\mathrm{e}}\right] \\
& q=-H \xi+\left(\sigma_{0}-\sigma_{\infty}\right)(1-\exp (-\delta \xi))
\end{aligned}
$$

(iv) Von-Mises yield condition

$$
\begin{aligned}
& \phi(\mathbf{s}, q):=\|\mathbf{s}\|-\sqrt{\frac{2}{3}}\left(\sigma_{0}-q\right) \leqslant 0 \\
& \text { (v) Associative flow rule } \\
& \mathscr{L}_{v} \overrightarrow{\mathbf{b}}=\gamma \frac{2}{3} \operatorname{tr}\left[\overline{\mathbf{b}}^{\mathrm{e}}\right] \mathbf{n}, \dot{\xi}=\gamma \sqrt{\frac{2}{3}}, \mathbf{n}=\mathbf{s} /\|\mathbf{s}\|
\end{aligned}
$$

(vi) Kuhn-Tucker loading/unloading conditions

$\gamma \geqslant 0, \quad \phi \leqslant 0, \quad \gamma \phi=0$

\section{(vii) Consistency condition}

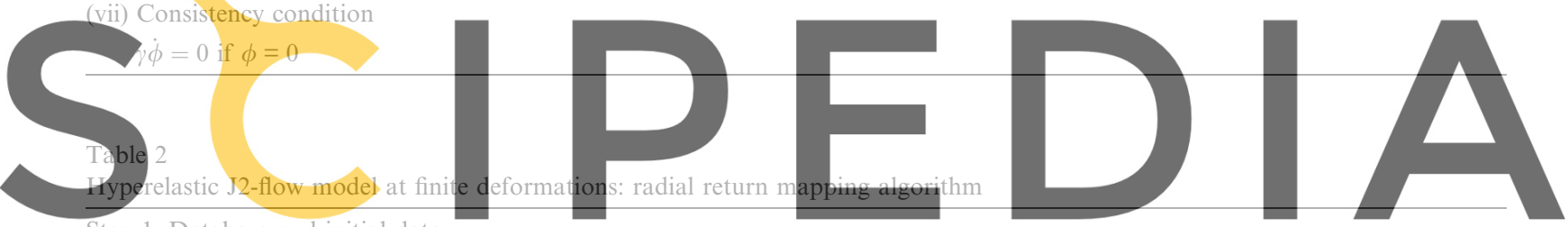

Step 1. Database and initial data

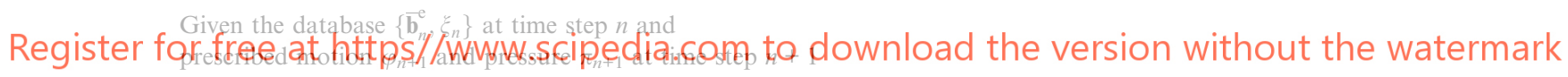

Step 2. Compute elastic predictor

$$
\begin{aligned}
& \overline{\mathbf{b}}_{n+1}^{e \text {,trial }}=\overline{\mathbf{f}}_{n+1} \overline{\mathbf{b}}_{n}^{\mathrm{e}} \overline{\mathbf{f}}_{n+1}^{\mathrm{I}}, \overline{\mathbf{f}}_{n+1}=\operatorname{det}\left[\mathbf{f}_{n+1}\right]^{-1 / 3} \mathbf{f}_{n+1}, \mathbf{f}_{n+1}=\mathbf{F}_{n+1} \mathbf{F}_{n}^{-1} \\
& \xi_{n+1}^{\text {trial }}=\xi_{n} \\
& \mathbf{s}_{n+1}^{\text {trial }}=\mu \operatorname{dev}\left[\overline{\mathbf{b}}_{n+1}^{e \text { trial }}\right], q_{n+1}^{\text {trial }}=-H \xi_{n+1}^{\text {trial }}+\left(\sigma_{0}-\sigma_{\infty}\right)\left(1-\exp \left(-\delta \xi_{n+1}^{\text {trial }}\right)\right) \\
& f_{n+1}^{\text {trial }}=\left\|\mathbf{s}_{n+1}^{\text {trial }}\right\|-\sqrt{\frac{2}{3}}\left(\sigma_{0}-q_{n+1}^{\text {trial }}\right)
\end{aligned}
$$

Step 3. Check for plastic loading

$$
\begin{aligned}
& \text { IF } f_{n+1}^{\text {trial }} \leqslant 0 \text { THEN } \\
& \text { Set }(\cdot)_{n+1}:=(\cdot)_{n+1}^{\text {trial }} \text { and EXIT } \\
& \text { END IF }
\end{aligned}
$$

Step 4. Radial return mapping

$$
\begin{aligned}
& \text { Set: } \bar{\mu}_{n+1}=\mu \bar{I}_{n+1}^{\mathrm{e}}, \bar{I}_{n+1}^{\mathrm{e}}:=\frac{1}{3} \operatorname{tr}\left[\overline{\mathbf{b}}_{n+1}^{e, \text { trial }}\right] \\
& \text { Compute: } \gamma_{n+1}=\frac{f_{n+1}^{\text {trial }} /\left(2 \bar{\mu}_{n+1}\right)}{1+H /\left(3 \bar{\mu}_{n+1}\right)}, \mathbf{n}_{n+1}:=\mathbf{s}_{n+1}^{\text {trial }} /\left\|\mathbf{s}_{n+1}^{\text {trial }}\right\| \\
& \mathbf{s}_{n+1}=\mathbf{s}_{n+1}^{\text {trial }}-2 \bar{\mu}_{n+1} \gamma_{n+1} \mathbf{n}_{n+1}, \quad \xi_{n+1}=\xi_{n+1}^{\text {trial }}+\sqrt{\frac{2}{3}} \gamma_{n+1}
\end{aligned}
$$

Step 5. Addition of elastic pressure

$$
\tau_{n+1}=\pi_{n+1} \mathbf{1}+\mathbf{s}_{n+1}
$$

Step 6. Update of intermediate configuration and database

$$
\overline{\mathbf{b}}_{n+1}^{\mathrm{e}}=\mathbf{s}_{n+1} / \mu+\bar{I}_{n+1}^{\mathrm{e}} \mathbf{1}
$$


Table 3

Hyperelastic J2-flow model at finite deformations: deviatoric consistent elastoplastic moduli

$\overline{\mathbf{c}_{n+1}^{\mathrm{dev}}}=\left(1-\beta_{1}\right) \mathbf{c}_{n+1}^{\mathrm{dev}, \mathrm{trial}}-2 \bar{\mu}\left(\beta_{3} \mathbf{n}_{n+1} \otimes \mathbf{n}_{n+1}+\beta_{4} \operatorname{sym}\left[\mathbf{n}_{n+1} \otimes \operatorname{dev}\left[\mathbf{n}_{n+1}^{2}\right]\right]\right)$

$\mathbf{c}_{n+1}^{\text {dev,trial }}=2 \bar{\mu}\left(\llbracket-\frac{1}{3} \mathbf{1} \otimes \mathbf{1}\right)-\frac{2}{3}\left\|\mathbf{s}_{n+1}^{\text {trial }}\right\|\left(\mathbf{n}_{n+1} \otimes \mathbf{1}+\mathbf{1} \otimes \mathbf{n}_{n+1}\right)$

$\beta_{0}=1+H /(3 \bar{\mu}), \beta_{1}:=2 \bar{\mu} \frac{\gamma_{n+1}}{\left\|\mathbf{s}_{n+1}^{\text {trial }}\right\|}, \beta_{2}:=\left(1-1 / \beta_{0}\right) \frac{2}{3} \frac{\left\|\mathbf{s}_{n+1}^{\text {trial }}\right\|}{\bar{\mu}} \gamma_{n+1}$

$\beta_{3}=1 / \beta_{0}-\beta_{1}+\beta_{2}, \quad \beta_{4}=\left(1 / \beta_{0}-\beta_{1}\right) \frac{\left\|\mathbf{s}_{n+1}^{\text {trial }}\right\|}{\bar{\mu}}$

$$
\begin{aligned}
\left.\left(\widetilde{\mathbf{s}}_{n+1}, \nabla^{s} \widetilde{\mathbf{v}}\right)\right|_{\Omega_{e}} & :=\left.\left(2 \widetilde{\mu}_{n+1} \operatorname{dev}\left[\nabla^{s} \widetilde{\mathbf{u}}_{n+1}\right], \nabla^{s} \widetilde{\mathbf{v}}\right)\right|_{\Omega_{e}}, \\
& :=\left.\left(\tau_{e, n+1}^{-1} \widetilde{\mathbf{u}}_{n+1}, \widetilde{\mathbf{v}}\right)\right|_{\Omega_{e}} \forall \widetilde{\mathbf{v}} \in \widetilde{\mathscr{V}}_{0},
\end{aligned}
$$

where the inverse of the (scalar) stabilization parameter $\tau_{e, n+1}$ is locally (at the element level) defined at time $n+1$ as

$$
\tau_{e, n+1}^{-1}=\frac{2 \widetilde{\mu}_{n+1}}{c h_{e}^{2}}
$$

where $c$ is a mesh-size independent constant to be determined numerically.

Remark 5. For elastic loading/unloading the subgrid scale shear modulus $\widetilde{\mu}_{n+1}$ is defined as $\widetilde{\mu}_{n+1}=\mu J_{h, n+1}^{-2 / 3}$.

\subsubsection{Subgrid scales}

Algebraic Subgrid Scales (ASGS) and Orthogonal Subgrid Scales (OSGS). Using (37), (38), (35) and (34) the following variational approximation for the subscales at the element level holds

$$
\left.\left(\widetilde{\mathbf{u}}_{n+1}, \widetilde{\mathbf{v}}\right)\right|_{\Omega_{e}}=\left.\left(\tau_{e, n+1}\left(J_{h, n+1} \nabla\left(J_{h, n+1}^{-1} \pi_{h, n+1}\right)+J_{h, n+1} \nabla \cdot\left(J_{h, n+1}^{-1} \mathbf{s}_{h, n+1}\right)+\mathbf{f}\right), \widetilde{\mathbf{v}}\right)\right|_{\Omega_{e}} \forall \widetilde{\mathbf{v}} \in \widetilde{\mathscr{V}}_{0}
$$

and the subgrid scales, at the element level, can be approximated as

$$
\widetilde{\mathbf{u}}_{n+1}=\tau_{e, n+1}\left(J_{h, n+1} \nabla\left(J_{h, n+1}^{-1} \pi_{h, n+1}\right)+J_{h, n+1} \nabla \cdot\left(J_{h, n+1}^{-1} \mathbf{s}_{h, n+1}\right)+\mathbf{f}\right)+\mathbf{v}_{h, \text { ort }},
$$

where $\mathbf{v}_{h, \text { ort }} \in \widetilde{\mathscr{W}}^{\perp}$ belongs to the orthogonal space of the subscales. Here different options are available to approximate the subscales, according to the expression chosen for $\mathbf{v}_{h \text {,ort }}$. Consider the following two options, leading to the so called Algebraic Subgrid Scales (ASGS) and Orthogonal Subgrid Scales (OSGS) methods, respectively.

\subsubsection{Algebraic subgrid scales method ( $A S G S$ )}

Within the ASGS method, we take $\mathbf{v}_{h \text {,ort }}=\mathbf{0}$ and the subgrid scale displacements, at the element level, are approximated as

$$
\widetilde{\mathbf{u}}_{n+1}=\tau_{e, n+1}\left(J_{h, n+1} \nabla\left(J_{h, n+1}^{-1} \pi_{h, n+1}\right)+J_{h, n+1} \nabla \cdot\left(J_{h, n+1}^{-1} \mathbf{s}_{h, n+1}\right)+\mathbf{f}\right) .
$$

Remark 6. Note that within the ASGS, the subscales can be viewed as proportional to the residual of the momentum balance equation provided by the finite element solution. 
Remark 7. Note that dropping the contributions to the subgrid scale displacements associated to the deviatoric Kirchhoff stress component and body forces per unit reference volume, the GLS method, originally proposed in the context of CFD by Hughes et al. [21,22] is recovered. Within the context of solid mechanics a stabilized formulation based on the GLS method has been used by Klaas et al. [24]. A comparison of the performance of OSGS and GLS methods can be found in Valverde et al. [44], Cervera et al. [7] or Oñate et al. [30]. On the other hand, for linear elements the finite element approximation of the Jacobian $J_{h, n+1}$ is constant within an element. Then, the approximation at the element level for the subscales using the GLS method leads to the following simple expression

$$
\widetilde{\mathbf{u}}_{n+1}=\tau_{e, n+1} \nabla \pi_{h, n+1} .
$$

Orthogonal subgrid scales method (OSGS). Within the OSGS method, we take $\mathbf{v}_{h, \text { ort }}=-\tau_{e, n+1} P_{h}\left(J_{h, n+1}\right.$ $\left.\nabla\left(J_{h, n+1}^{-1} \pi_{h, n+1}\right)+J_{h, n+1} \nabla \cdot\left(J_{h, n+1}^{-1} \mathbf{s}_{h, n+1}\right)+\mathbf{f}\right)$, where $P_{h}(\cdot)$ represents the $L_{2}$ projection onto the finite element space $\mathscr{W}_{h}$, and the subgrid scale displacements, at the element level, are approximated as

$$
\widetilde{\mathbf{u}}_{n+1}=\tau_{e, n+1} P_{h}^{\perp}\left(J_{h, n+1} \nabla\left(J_{h, n+1}^{-1} \pi_{h, n+1}\right)+J_{h, n+1} \nabla \cdot\left(J_{h, n+1}^{-1} \mathbf{s}_{h, n+1}\right)+\mathbf{f}\right),
$$

where $P_{h}^{\perp}(\cdot)=(\cdot)-P_{h}(\cdot)$ is the $L_{2}$ orthogonal projection onto $\mathscr{W}_{h}^{\perp}$.

Remark 8. Note that within the OSGS we are implicitly considering that $\widetilde{\mathscr{W}} \approx \mathscr{W}_{h}^{\perp}$, i.e., we are taking the orthogonal space to the finite element solution space as space of the subscales. Then, the subscales can be viewed as proportional to the orthogonal projection to the finite element space of the residual of the momentum balance equation provided by the finite element solution.

Remark 9. We will assume that the body forces per unit reference volume belong to the finite element solution space, i.e., $P_{h}^{\perp}(\mathbf{f})=\mathbf{0}$, and we will neglect the contribution arising from the deviatoric Kirchhoff stress component, e.g., we will assume that $P_{h}^{\perp}\left(J_{h, n+1} \nabla \cdot\left(J_{h, n+1}^{-1} \mathbf{s}_{h, n+1}\right)\right)=\mathbf{0}$ holds. On the other hand, for linear elements the finite element approximation of the Jacobian $J_{h, n+1}$ is constant within an element. Then, the approximation at the element level for the subscales using the OSGS method leads to the following simple expression

$$
\widetilde{\mathbf{u}}_{n+1}=\tau_{e, n+1} P_{h}^{\perp}\left(\nabla \pi_{h, n+1}\right) \text {. }
$$

In this work we will mainly adopt the OSGS method as the variational multiscale stabilization method.

\subsection{Discrete stabilized variational form}

The goal now is to introduce the approximation found for the subscales using the OSGS method into the finite-dimensional variational problem (31), (32). Using the stress split (27), integrating by parts the last term of the left-hand side of (32) within each element and neglecting the interelement boundary terms or assuming that the subgrid scales vanish at the element boundaries (as for bubble enhancements), the variational stabilized Eqs. (31) and (32) can be written as

$$
\begin{aligned}
& \left(\pi_{h, n+1}, \nabla \cdot \mathbf{v}_{h}\right)+\left(\mathbf{s}_{h, n+1}, \nabla^{s} \mathbf{v}_{h}\right)+\left.\sum_{e=1}^{n_{\mathrm{elm}}}\left(\widetilde{\mathbf{s}}_{n+1}, \nabla^{s} \mathbf{v}_{h}\right)\right|_{\Omega_{e}}=l_{n+1}\left(\mathbf{v}_{h}\right) \quad \forall \mathbf{v}_{h} \in \mathscr{V}_{h, 0}, \\
& \left(\log J_{h, n+1}-\frac{1}{\kappa} \pi_{h, n+1}, q_{h}\right)-\left.\sum_{e=1}^{n_{\mathrm{elm}}}\left(\widetilde{\mathbf{u}}_{h+1}, J_{h, n+1} \nabla\left(J_{h, n+1}^{-1} q_{h}\right)\right)\right|_{\Omega_{e}}=0 \quad \forall q_{h} \in \mathscr{Q}_{h} .
\end{aligned}
$$


Using the constitutive equation (37), integrating by parts the last term of the left-hand side of (31) within each element and neglecting the interelement boundary terms or assuming that subgrid scales vanish at the element boundaries (as for bubble enhancements), the last term of the left-hand side of (31) may be written at the element level as

$$
\begin{aligned}
\left(\widetilde{\mathbf{s}}_{n+1}, \nabla^{s} \mathbf{v}_{h}\right) & =\left(2 \widetilde{\mu}_{n+1} \operatorname{dev}\left[\nabla^{s} \widetilde{\mathbf{u}}_{n+1}\right], \nabla^{s} \mathbf{v}_{h}\right)=\left(\nabla^{s} \widetilde{\mathbf{u}}_{n+1}, 2 \widetilde{\mu}_{n+1} \operatorname{dev}\left[\nabla^{s} \mathbf{v}_{h}\right]\right) \\
& =-\left(\widetilde{\mathbf{u}}_{n+1}, J_{h, n+1} \nabla \cdot\left(J_{h, n+1}^{-1} 2 \widetilde{\mu}_{n+1} \operatorname{dev}\left[\nabla^{s} \mathbf{v}_{h}\right]\right)\right)
\end{aligned}
$$

and, substituting this result into the above variational forms, we obtain

$$
\begin{aligned}
& \left(\pi_{h, n+1}, \nabla \cdot \mathbf{v}_{h}\right)+\left(\mathbf{s}_{h, n+1}, \nabla^{s} \mathbf{v}_{h}\right)-\left.\sum_{e=1}^{n_{\mathrm{elm}}}\left(\widetilde{\mathbf{u}}_{n+1}, J_{h, n+1} \nabla \cdot\left(J_{h, n+1}^{-1} 2 \widetilde{\mu}_{n+1} \operatorname{dev}\left[\nabla^{s} \mathbf{v}_{h}\right]\right)\right)\right|_{\Omega_{e}}=l_{n+1}\left(\mathbf{v}_{h}\right) \quad \forall \mathbf{v}_{h} \in \mathscr{V}_{h, 0} \\
& \left(\log J_{h, n+1}-\frac{1}{\kappa} \pi_{h, n+1}, q_{h}\right)-\left.\sum_{e=1}^{n_{\mathrm{elm}}}\left(\widetilde{\mathbf{u}}_{n+1}, J_{h, n+1} \nabla\left(J_{h, n+1}^{-1} q_{h}\right)\right)\right|_{\Omega_{e}}=0 \quad \forall q_{h} \in \mathscr{Q}_{h}
\end{aligned}
$$

Remark 10. Note that for linear elements $J_{h, n+1}$ and $\operatorname{dev}\left[\nabla^{s} \mathbf{v}_{h}\right]$ are constant within the elements. Then $\nabla \cdot\left(J_{h, n+1}^{-1} \operatorname{dev}\left[\nabla^{s} \mathbf{v}_{h}\right]\right)=0$ and $J_{h, n+1} \nabla\left(J_{h, n+1}^{-1} q_{h}\right)=\nabla q_{h}$. Furthermore, the last term of the left-hand side of (41) will be neglected, i.e. $\nabla \cdot\left(J_{h, n+1}^{-1} 2 \widetilde{\mu}_{n+1} \operatorname{dev}\left[\nabla^{s} \mathbf{v}_{h}\right]\right)=0$.

Then, using the above Remark and introducing the approximation for the subscales given by (40), the stabilized variational formulation can be written as: find $\left(\mathbf{u}_{h, n+1}, \pi_{h, n+1}\right) \in \mathscr{W}_{h}$ such that

$$
\begin{aligned}
& \left(\pi_{h, n+1}, \nabla \cdot \mathbf{v}_{h}\right)+\left(\mathbf{s}_{h, n+1}, \nabla^{s} \mathbf{v}_{h}\right)=l_{n+1}\left(\mathbf{v}_{h}\right) \quad \forall \mathbf{v}_{h} \in \mathscr{V}_{h, 0}, \\
& \left(\log J_{h, n+1}-\frac{1}{\kappa} \pi_{h, n+1}, q_{h}\right)-\left.\sum_{e=1}^{n_{\mathrm{elm}}}\left(\tau_{e, n+1} P_{h}^{\perp}\left(\nabla \pi_{h, n+1}\right), \nabla q_{h}\right)\right|_{\Omega_{e}}=0 \quad \forall q_{h} \in \mathscr{V}_{h} .
\end{aligned}
$$

Remark 11. Note that (43), (44) correspond to the time discrete counterpart of the discrete stabilized variational problem defined as: find $\mathbf{U}_{h} \in \mathscr{W}_{h}$ such that for any $\mathbf{V}_{h} \in \mathscr{W}_{h, 0}$

$$
B_{\text {stab }}\left(\mathbf{U}_{h} \mathbf{V}_{h}\right)=L_{\text {stab }}\left(\mathbf{V}_{h}\right) \text {, }
$$

where the OSGS stabilized (mesh-dependent) forms $B_{\text {stab }}\left(\mathbf{U}_{h}, \mathbf{V}_{h}\right)$ and $L_{\text {stab }}\left(\mathbf{V}_{h}\right)$ can be written as

$$
\begin{aligned}
& B_{\text {stab }}\left(\mathbf{U}_{h}, \mathbf{V}_{h}\right):=B\left(\mathbf{U}_{h}, \mathbf{V}_{h}\right)-\left.\sum_{e=1}^{n_{\text {elm }}}\left(\tau_{e, n+1} P_{h}^{\perp}\left(\nabla \pi_{h, n+1}\right), \nabla q_{h}\right)\right|_{\Omega_{e}}, \\
& L_{\text {stab }}\left(\mathbf{V}_{h}\right):=L\left(\mathbf{V}_{h}\right) .
\end{aligned}
$$

Set $\Pi_{h, n+1}:=P_{h}\left(\nabla \pi_{h, n+1}\right)$ as the projection of the Kirchhoff pressure gradient onto the finite element space $\mathscr{W}_{h}$. Let $\Upsilon=\mathbf{H}^{1}$ and $\Upsilon_{h} \subset \Upsilon$ be the space of the Kirchhoff pressure gradient projection and its finite element associated subspace, respectively. Then, taking $\Pi_{h, n+1} \in \Upsilon_{h}$ as an additional independent continuous variable, the orthogonal projection of the discrete Kirchhoff pressure gradient can be written as $P_{h}^{\perp}\left(\nabla \pi_{h, n+1}\right):=\nabla \pi_{h, n+1}-\Pi_{h, n+1}$ and the following variational form holds:

$$
\left.\left(\nabla \pi_{h, n+1}, \eta_{h}\right)\right|_{\Omega_{e}}-\left.\left(\Pi_{h, n+1}, \eta_{h}\right)\right|_{\Omega_{e}}=0 \quad \forall \eta_{h} \in \mathscr{V}_{h, 0} .
$$


Finally, introducing the discrete Kirchhoff pressure gradient projection as a third variable, the stabilized variational problem can be written as: find $\left(\mathbf{u}_{h, n+1}, \pi_{h, n+1}, \Pi_{h, n+1}\right) \in \mathscr{V}_{h} \times \mathscr{Q}_{h} \times \Upsilon_{h}$ such that

$$
\begin{aligned}
& \left(\pi_{h, n+1}, \nabla \cdot \mathbf{v}_{h}\right)+\left(\mathbf{s}_{h, n+1}, \nabla^{s} \mathbf{v}_{h}\right)=l_{n+1}\left(\mathbf{v}_{h}\right) \quad \forall \mathbf{v}_{h} \in \mathscr{V}_{h, 0}, \\
& \left(\log J_{h, n+1}-\frac{1}{\kappa} \pi_{h, n+1}, q_{h}\right)-\left.\sum_{e=1}^{n_{\mathrm{elm}}}\left(\tau_{e, n+1}\left(\nabla \pi_{h, n+1}-\Pi_{h, n+1}\right), \nabla q_{h}\right)\right|_{\Omega_{e}}=0 \quad \forall q_{h} \in \mathscr{Q}_{h}, \\
& \left.\sum_{e=1}^{n_{\mathrm{elm}}}\left(\nabla \pi_{h, n+1}-\Pi_{h, n+1}, \eta_{h}\right)\right|_{\Omega_{e}}=0 \quad \forall \eta_{h} \in \mathscr{V}_{h, 0} .
\end{aligned}
$$

Remark 12. Note that within the OSGS method the variational stabilization term at the element level $-\left.\left(\tau_{e, n+1}\left(\nabla \pi_{h, n+1}-\Pi_{h, n+1}\right), \nabla q_{h}\right)\right|_{\Omega_{e}}$ is proportional to the difference between the continuous (projected) and the discontinuous (elemental) Kirchhoff pressure gradients, while within the GLS method the stabilization term is proportional to the Kirchhoff pressure gradient itself. Therefore, in both cases the stabilization terms decreases very rapidly upon mesh refinement but for the OSGS method this happens at a greater rate, and then the OSGS method exhibit a better accuracy, robustness and less sensitivity to the stabilization parameter than the GLS method as it is shown in Valverde et al. [44], Cervera et al. [7] or Oñate et al. [30].

Remark 13. Note that in the final stabilized variational problem there is only a remaining stabilization term which appears in (46), while the (45) remains the same as for the original problem. On the other hand, a further variable has been introduced. However, as it is shown in the next section, this drawback can easily be overcome to get a computational robust and efficient procedure.

Remark 14. To gain further insight on the OSGS stabilization technique, note that the resulting stabilized variational equations could be viewed (assuming that the stabilization parameter is constant) as the variational form of the following stabilized continuous (strong form) equations:

$$
\begin{aligned}
& J \nabla\left(J^{-1} \pi\right)+J \nabla \cdot\left(J^{-1} \mathbf{s}(\mathbf{u})\right)+\mathbf{f}=\mathbf{0} \text { in } \Omega, \\
& \frac{1}{\kappa} \pi-\log J-\tau\left(\nabla^{2} \pi-\nabla \cdot \Pi\right)=0 \text { in } \Omega, \\
& \nabla \pi-\Pi=\mathbf{0} \text { in } \Omega
\end{aligned}
$$

with the appropriate boundary conditions.

\section{Computational and implementation aspects}

From the computational efficiency point of view, the drawback related to the introduction of a new variable field inherent to the OSGS can be easily overcome to end up with a computational robust and efficient solution algorithm. A convenient staggered solution method can be obtained by a slight modification of the problem defined by the stabilized variational system of Eqs. (45)-(47), in which we keep fixed the Kirchhoff pressure gradient projection field $\Pi_{h, n}$ at the last converged time step $t_{n}$ while solving for the displacement and Kirchhoff pressure fields $\mathbf{u}_{h, n+1}$ and $\pi_{h, n+1}$, respectively, at time $t_{n+1}$. The update of the Kirchhoff pressure gradient projection field is performed in a second step. Therefore the modified algorithmic stabilized variational problem can be written as a two-step problem defined as follows. 
Problem 1. Given $\Pi_{h, n} \in \Upsilon_{h}$ find $\left(\mathbf{u}_{h, n+1}, \pi_{h, n+1}\right) \in \mathscr{V}_{h} \times \mathscr{Q}_{h}$ such that

$$
\begin{aligned}
& \left(\pi_{h, n+1}, \nabla \cdot \mathbf{v}_{h}\right)+\left(\mathbf{s}_{h, n+1}, \nabla^{s} \mathbf{v}_{h}\right)=l_{n+1}\left(\mathbf{v}_{h}\right) \quad \forall \mathbf{v}_{h} \in \mathscr{V}_{h, 0}, \\
& \left(\log J_{h, n+1}-\frac{1}{\kappa} \pi_{h, n+1}, q_{h}\right)-\left.\sum_{e=1}^{n_{\mathrm{elm}}}\left(\tau_{e, n}\left(\nabla_{n} \pi_{h, n+1}-\Pi_{h, n}\right), \nabla_{h} q_{h}\right)\right|_{\Omega_{e}}=0 \quad \forall q_{h} \in \mathscr{Q}_{h},
\end{aligned}
$$

where $\nabla_{n}$ denotes the spatial gradient with respect to the previous converged configuration at time step $t_{n}$. Note also that the stabilization parameter $\tau_{e, n}$ is evaluated at time step $t_{n}$.

The solution of Problem 1 is obtained using a Newton-Raphson incremental iterative algorithm. The resulting linearized variational system of equations at time step $n+1$, iteration $i+1$, can be written as: given $\Pi_{h, n} \in \Upsilon_{h}$, find $\left(\Delta \mathbf{u}_{h, n+1}^{(i)}, \Delta \pi_{h, n+1}^{(i)}\right) \in \mathscr{V}_{h} \times \mathscr{Q}_{h}$ such that

$$
\begin{aligned}
& \left(\Delta \pi_{h, n+1}^{(i)}, \nabla \cdot \mathbf{v}_{h}\right)+\left(\left(\mathbf{c}_{h, n+1}^{\operatorname{dev}(i)}-2 \pi_{h, n+1}^{(i)} \mathbf{I}\right): \nabla^{s} \Delta \mathbf{u}_{h, n+1}^{(i)}, \nabla^{s} \mathbf{v}_{h}\right)+\left(\nabla \Delta \mathbf{u}_{h, n+1}^{(i)}\left(\pi_{h, n+1}^{(i)} \mathbf{1}+\mathbf{s}_{h, n+1}^{(i)}\right), \nabla \mathbf{v}_{h}\right) \\
& \quad=-R_{u}\left(\mathbf{u}_{h, n+1}^{(i)}, \pi_{h, n+1}^{(i)} ; \mathbf{v}_{h}\right) \quad \forall \mathbf{v}_{h} \in \mathscr{V}_{h, 0} \\
& \left(\nabla \cdot \Delta \mathbf{u}_{h, n+1}^{(i)}-\frac{1}{\kappa} \Delta \pi_{h, n+1}^{(i)}, q_{h}\right)-\left.\sum_{e=1}^{n_{\mathrm{elm}}}\left(\tau_{e, n} \nabla_{n} \Delta \pi_{h, n+1}^{(i)}, \nabla_{n} q_{h}\right)\right|_{\Omega_{e}}=-R_{\pi}\left(\mathbf{u}_{h, n+1}^{(i)}, \pi_{h, n+1}^{(i)} ; q_{h}\right) \quad \forall q_{h} \in \mathscr{Q}_{h},
\end{aligned}
$$

where $R_{u}\left(\mathbf{u}_{h, n+1}^{(i)}, \pi_{h, n+1}^{(i)} ; \mathbf{v}_{h}\right)$ and $R_{\pi}\left(\mathbf{u}_{h, n+1}^{(i)}, \pi_{h, n+1}^{(i)} ; q_{h}\right)$ denote the variational residual equations at time step $n+1$, iteration $i$, and are defined as

$$
\begin{aligned}
& R_{u}\left(\mathbf{u}_{h, n+1}^{(i)}, \pi_{h, n+1}^{(i)} ; \mathbf{v}_{h}\right):=\left(\pi_{h, n+1}^{(i)}, \nabla \cdot \mathbf{v}_{h}\right)+\left(\mathbf{s}_{h, n+1}^{(i)}, \nabla^{s} \mathbf{v}_{h}\right)-l_{n+1}\left(\mathbf{v}_{h}\right) \quad \forall \mathbf{v}_{h} \in \mathscr{V}_{h, 0}, \\
& R_{\pi}\left(\mathbf{u}_{h, n+1}^{(i)}, \pi_{h, n+1}^{(i)} ; q_{h}\right):=\left(\log J_{h, n+1}^{(i)}-\frac{1}{\kappa} \pi_{h, n+1}^{(i)}, q_{h}\right)-\left.\sum_{e=1}^{n_{\mathrm{elm}}}\left(\tau_{e, n}\left(\nabla_{n} \pi_{h, n+1}^{(i)}-\Pi_{h, n}\right), \nabla_{n} q_{h}\right)\right|_{\Omega_{e}} \quad \forall q_{h} \in \mathscr{V}_{h} .
\end{aligned}
$$

Problem 2. Given $\left(\mathbf{u}_{h, n+1}, \pi_{h, n+1}\right) \in \mathscr{V}_{h} \times \mathscr{Q}_{h}$, find $\Pi_{h, n+1} \in \Upsilon_{h}$ such that

$$
\left.\sum_{e=1}^{n_{\mathrm{elm}}}\left(\nabla \pi_{h, n+1}-\Pi_{h, n+1}, \eta_{h}\right)\right|_{\Omega_{e}}=0 \quad \forall \eta_{h} \in \mathscr{V}_{h, 0} .
$$

\subsection{Finite element projection}

Once the finite element discretization has been introduced, the matrix form of the algebraic system resulting from the variational Problem 1 takes the form

$$
\begin{aligned}
& \mathbb{K}_{T_{n+1}^{(i)}}^{(i)} \mathbb{U}_{n+1}^{(i)}+\mathbb{G}_{n+1}^{(i)} \Delta \mathbb{P}_{n+1}^{(i)}=-\mathbb{R}_{u, n+1}^{(i)} \\
& \mathbb{G}_{n+1}^{(i) \mathrm{T}} \Delta \mathbb{U}_{n+1}^{(i)}-\left(\mathbb{M}_{p}+\mathbb{L}_{\tau, n}\right) \Delta \mathbb{P}_{n+1}^{(i)}=-\mathbb{R}_{\pi, n+1}^{(i)},
\end{aligned}
$$

where $\mathbb{K}_{T_{n+1}}^{(i)}, \mathbb{G}_{n+1}^{(i)}, \mathbb{M}_{p}$ and $\mathbb{L}_{\tau, n}$ denote the matrices arising from the finite element projection of the last three terms of the left-hand side of (48), the first term of the left-hand side of (48), the first term of the left-hand side of (49) and the third term of the left-hand side of (49), respectively, $\mathbb{R}_{u, n+1}^{(i)}$ and $\mathbb{R}_{\pi, n+1}^{(i)}$ denote the vectors arising from the finite element projection of the variational residuals $R_{u}\left(\mathbf{u}_{h, n+1}^{(i)}, \pi_{h, n+1}^{(i)} ; \mathbf{v}_{h}\right)$ and $R_{\pi}\left(\mathbf{u}_{h, n+1}^{(i)}, \pi_{h, n+1}^{(i)} ; q_{h}\right)$, respectively, and $\Delta \mathbb{U}_{n+1}^{(i)}:=\mathbb{U}_{n+1}^{(i+1)}-\mathbb{U}_{n+1}^{(i)}$ and $\Delta \mathbb{P}_{n+1}^{(i)}:=\mathbb{P}_{n+1}^{(i+1)}-\mathbb{P}_{n+1}^{(i)}$ denote the increments of nodal displacement and Kirchhoff pressure unknowns, respectively. 
Remark 15. It should be noted that the resulting system of equations is symmetric. Block-symmetry arises as a result of the specific volumetric part of the stored energy function considered, due to the fact that $\left(J U^{\prime}(J)\right)^{\prime}=\kappa J^{-1}$ and $D J \cdot \Delta \mathbf{u}=J \nabla \cdot \Delta \mathbf{u}$.

Once the finite element discretization has been introduced, the matrix form of the algebraic system resulting from the variational Problem 2 takes the form

$$
\Pi_{n+1}=\mathbb{M}_{\tau, n+1}^{-1} \mathbb{G}_{\tau, n+1} \mathbb{P}_{n+1},
$$

where $\mathbb{M}_{\tau, n+1}$ and $\mathbb{G}_{\tau, n+1}$ denote the matrices arising from the stabilized displacement mass-like term and spatial gradient operator, respectively, and $\mathbb{P}_{n+1}$ and $\Pi_{n+1}$ denote the vectors of Kirchhoff pressure and Kirchhoff pressure gradient projection nodal unknowns at time step $n+1$, respectively. A lumped approximation to the stabilized mass-like matrix $\mathbb{M}_{\tau, n+1}$ would lead to a simple direct computation of the nodal Kirchhoff pressure gradient projection unknowns.

\subsection{Finite element matrices at element level}

Typical element entries $(\cdot)^{A B}$ corresponding to nodes $A$ and $B$ for the above matrices and $(\cdot)^{A}$ corresponding to node $A$ for the above residual vectors take the form

$$
\begin{aligned}
&\left.\left.\mathbb{K}\right|_{T}\right|_{\Omega_{e}} ^{A B}=\int_{\Omega_{e}}\left(\mathbf{B}_{u}^{A}\right)^{\mathrm{T}}\left(\mathbf{C}_{T}^{\mathrm{dev}}-2 \pi \mathbf{I}\right) \mathbf{B}_{u}^{B} \mathrm{~d} V_{0}+\int_{\Omega_{e}}\left(\nabla N_{u}^{A}\right)^{\mathrm{T}} \tau \nabla N_{u}^{B} \mathrm{~d} V_{0} \mathbf{1}, \\
&\left.\mathbb{G}\right|_{\Omega_{e}} ^{A B}=\int_{\Omega_{e}} \nabla N_{u}^{A} N_{\pi}^{B} \mathrm{~d} V_{0}, \\
&\left.\mathbb{M}_{p}\right|_{\Omega_{e}} ^{A B}=\int_{\Omega_{e}} \frac{1}{\kappa} N_{\pi}^{A} N_{\pi}^{B} \mathrm{~d} V_{0}, \\
&\left.\mathbb{L}_{\tau}\right|_{\Omega_{e}} ^{A B}=\int_{\Omega_{e}} \tau_{e}\left(\nabla N_{\pi}^{A}\right)^{\mathrm{T}} \nabla N_{\pi}^{B} \mathrm{~d} V_{0}, \\
&\left.\mathbb{G}_{\tau}\right|_{\Omega_{e}} ^{A B}=\int_{\Omega_{e}} \tau_{e} \nabla N_{\pi}^{A} N_{\pi}^{B} \mathrm{~d} V_{0}, \\
&\left.\mathbb{M}_{\tau}\right|_{\Omega_{e}} ^{A B}=\int_{\Omega_{e}} \tau_{e} N_{\Pi}^{A} N_{\Pi}^{B} \mathrm{~d} V_{0} \mathbf{1}, \\
&\left.\mathbb{R}_{u}\right|_{\Omega_{e}} ^{A}=\int_{\Omega_{e}}\left(\mathbf{B}_{u}^{A}\right)^{\mathrm{T}} \tau \mathrm{d} V_{0}-\int_{\Omega_{e}} N_{u}^{A} \mathbf{f} \mathrm{d} V_{0}-\int_{\partial \Omega_{e} \cap \partial \Omega_{t}} N_{u}^{A} \overline{\mathbf{t}}^{N} \mathrm{~d} S_{0}, \\
&\left.\mathbb{R}_{\pi}\right|_{\Omega_{e}} ^{A}=\int_{\Omega_{e}} N_{\pi}^{A}\left(\log J-\frac{1}{\kappa} \pi\right) \mathrm{d} V_{0}-\int_{\Omega_{e}} \tau_{e, n}\left(\nabla_{n} N_{\pi}^{A}\right)^{\mathrm{T}}\left(\nabla_{n} \pi-\Pi_{h, n}\right) \mathrm{d} V_{0},
\end{aligned}
$$

where $N_{u}^{A}, N_{\pi}^{A}$ and $N_{\Pi}^{A}$ denote the interpolation shape functions at node $A$ for the displacement, Kirchhoff pressure and Kirchhoff pressure gradient projection fields, respectively, $\nabla N_{u}^{A}$ and $\nabla N_{\pi}^{A}$ denote the gradient of the interpolation shape functions at node $A$ for the displacement and Kirchhoff pressure fields, respectively, and $\mathbf{B}_{u}^{A}$ denote the interpolation matrix at node $A$ for the symmetric spatial gradient field.

\section{Computational simulations}

The formulation presented in the preceeding sections is illustrated below in a number of computational simulations. Performance of the OSGS stabilized formulation is shown using triangular finite elements discretizations for $2 \mathrm{D}$ plane strain problems and tetrahedral finite elements discretizations for $3 \mathrm{D}$ problems. A 
plane strain tensile test of a rectangular bar, the upsetting of a 3D block and the necking of a 3D bar at finite strains are considered. The Newton-Raphson method, combined with a line search procedure, is used to solve the non-linear system of equations arising from the spatial and temporal discretization of the stabilized variational problem. Simulations have been performed with an enhanced version of the finite element program COMET [5] developed by the authors at the International Center for Numerical Methods in Engineering (CIMNE). Pre- and post-processing has been performed using GiD [18], also developed at CIMNE.

\subsection{Plane strain tensile test of a rectangular bar}

This example is concerned with the plane strain tensile test of a rectangular bar and has been studied by a number of authors as a localization problem using different softening behaviours [37]. The specimen considered here has a width of $12.826 \mathrm{~mm}$ and a length of $53.334 \mathrm{~mm}$ and is subjected to a tensile test under ideal plane strain loading conditions. In order to trigger the necking, we consider an initial geometric imperfection in the form of a reduction of the width from its initial value at the top to $0.982 \%$ of this value at the center of the specimen. Fig. 1 shows the (doubled symmetrized) quadrilateral (231 nodes and 200 elements) and (vertical symmetrized) triangular (803 nodes and 1464 elements) mesh discretizations used in the simulations. Loading is imposed using displacement control. A maximum vertical displacement of $5 \mathrm{~mm}$ is applied at the top and bottom edges of the bar. The material model is assumed to be elastoplastic at finite deformations. Elastic response is given by the stored energy (1). Plastic response is modeled by a J2-flow theory with linear and saturation isotropic hardening. Material properties are summarized in Table 4. The following finite elements have been considered in the simulations: (a) Q1/P0 mixed bilinear displacements/constant pressure quadrilateral element; (b) P1 standard irreductible linear displacements triangular

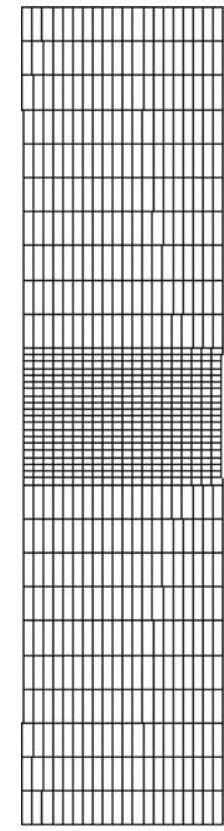

a

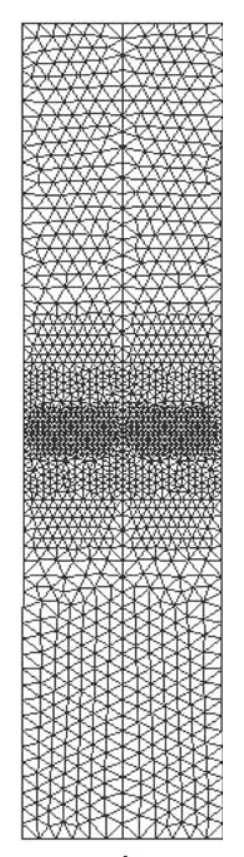

b

Fig. 1. Plane strain tensile test of a rectangular bar. Finite element discretization of the specimen: (a) quadrilateral mesh and (b) triangular mesh. 
Table 4

Plane strain tensile test of a rectangular bar, material properties

\begin{tabular}{llcc}
\hline Shear modulus & $\mu$ & 80.1938 & $\mathrm{GPa}$ \\
Bulk modulus & $\kappa$ & 164.206 & $\mathrm{GPa}$ \\
Initial flow stress & $\sigma_{0}$ & 0.45 & $\mathrm{GPa}$ \\
Residual flow stress & $\sigma_{\infty}$ & 0.715 & $\mathrm{GPa}$ \\
Linear hardening coefficient & $H$ & 0.12924 & $\mathrm{GPa}$ \\
Saturation hardening exponent & $\delta$ & 16.93 & \\
\hline
\end{tabular}

element; (c) P1/P1 mixed linear displacements/linear pressure triangular element; (d) P1/P1 OSGS stabilized mixed linear displacements/linear pressure triangular element.

Fig. 2 shows the deformed meshes obtained at the final stage of the simulation for each one of the elements considered. Note that the P1 irreductible linear displacements formulation does not allow to fully capture the necking, while in all the other formulations necking develops properly.

Figs. 3 and 4 collect the numerical results obtained at the final stage of the simulation for the equivalent plastic strain and the Kirchhoff pressure distributions for each one of the elements considered. Fig. 3 clearly shows again that the P1 irreductible linear displacements formulation can not capture properly the development of the necking, giving an incorrect distribution of the equivalent plastic strain in this area. The distributions of the equivalent plastic strain given by all the other elements formulations considered are similar. Fig. 4 clearly shows once again the unability of the P1 irreductible linear displacements formulation to deal with incompressible or quasi-incompressible problems, displaying lack of stability as high spurious

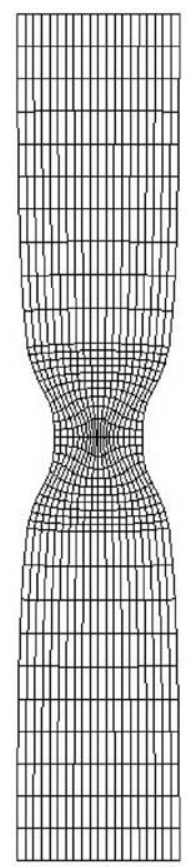

a

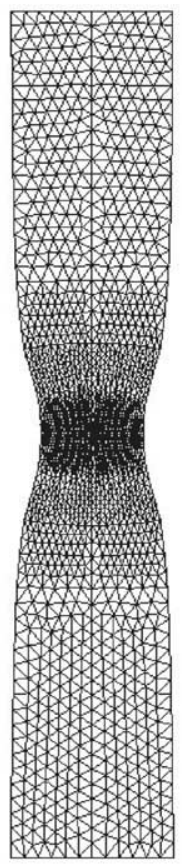

b

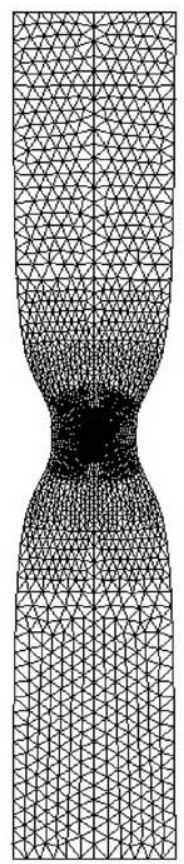

c

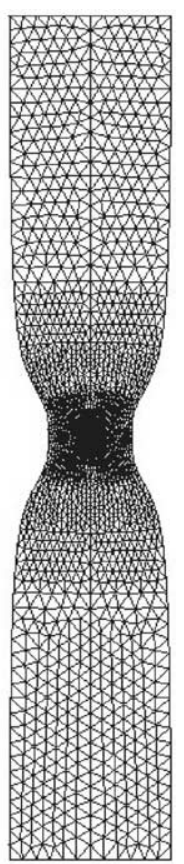

d

Fig. 2. Plane strain tensile test of a rectangular bar. Deformed meshes: (a) Q1/P0 bilinear displacement/constant pressure quadrilateral element; (b) P1 irreductible displacements triangular element; (c) P1/P1 standard mixed linear displacements/linear pressure triangular element and (d) P1/P1 OSGS stabilized mixed linear displacements/linear pressure triangular element. 


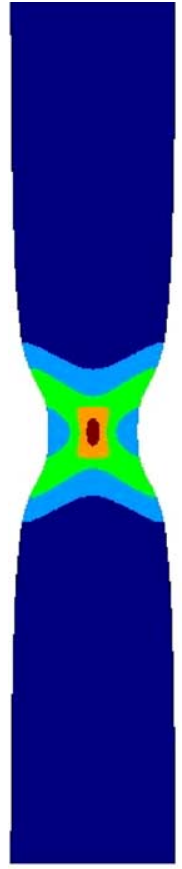

a

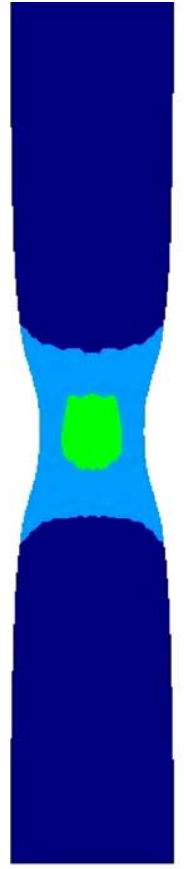

b

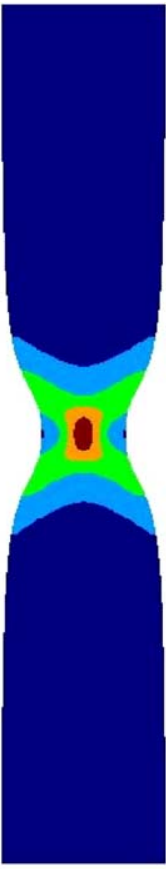

C

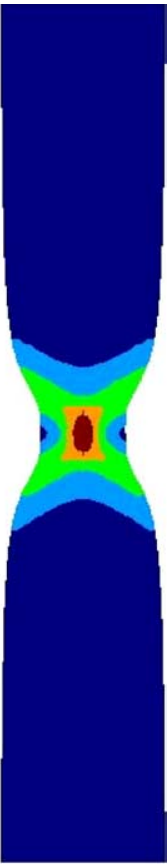

d

Fig. 3. Plane strain tensile test of a rectangular bar. Equivalent plastic strain distribution: (a) Q1/P0 bilinear displacement/constant pressure quadrilateral element; (b) P1 irreductible displacements triangular element; (c) P1/P1 standard mixed linear displacements/ linear pressure triangular element and (d) P1/P1 OSGS stabilized mixed linear displacements/linear pressure triangular element.

oscillations of the pressure that entirely pollute the solution. This lack of stability and uncontrollable pressure oscillations are not removed using a P1/P1 mixed linear displacements/linear pressure triangular element, while stability is attained and oscillations of the pressure are fully removed using the proposed P1/P1 OSGS stabilized mixed linear displacements/linear pressure triangular elements.

Figs. 5 and 6 show the plots obtained for the necking displacement and force versus time, respectively, for the P1 irreductible linear displacements triangular element, P1/P1 mixed linear displacements/linear pressure triangular element, Q1/P0 mixed bilinear displacements/constant pressure quadrilateral element and P1/P1 OSGS stabilized mixed linear displacements/linear pressure triangular element. The results show again the unability of the P1 irreductible linear displacements triangular element to capture the development of the necking.

\subsection{Upsetting of a $3 D$ block}

In this example the upsetting of a 3D block is considered. A 3D steel block of $0.85 \times 0.85 \times 0.60 \mathrm{~m}$ is subjected to compression by prescribing the vertical displacement of the top surface up to $15 \%$ of its initial height. Fig. 7 shows an external view of a quarter part of the initial and deformed geometry discretized using a mesh of linear tetrahedra with 1050 nodes. Boundary conditions are such that horizontal displacements at the top surface and displacements at the bottom surface are prescribed to zero. The material model is assumed to be elastoplastic at finite deformations. Compressible elastic response is considered. Plastic response is modeled by a J2-flow theory with exponential saturation isotropic hardening. Material properties are summarized in Table 5. 


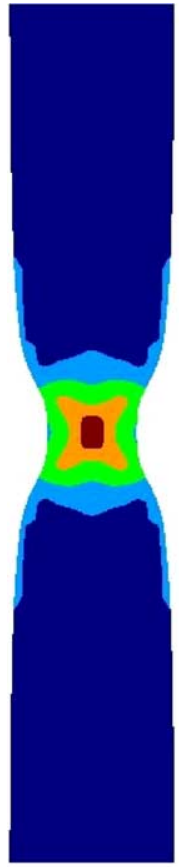

a

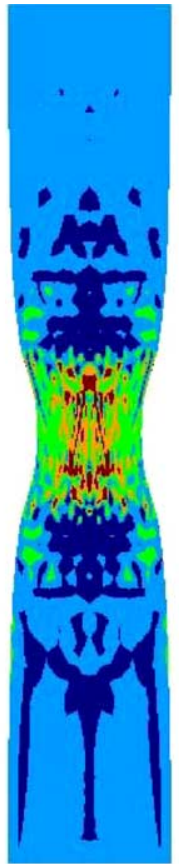

b

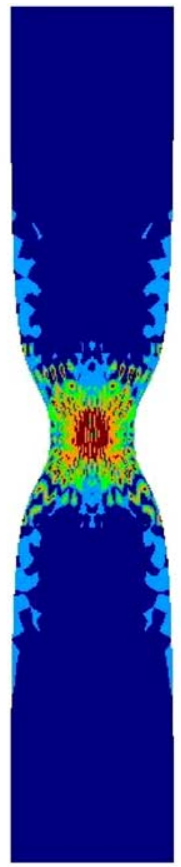

c

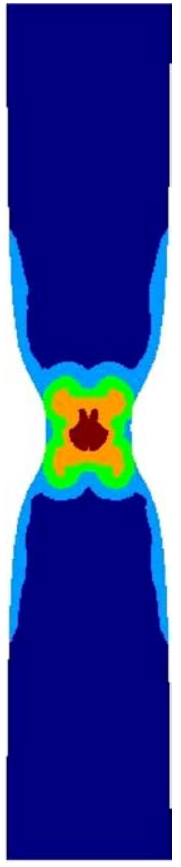

d

Fig. 4. Plane strain tensile test of a rectangular bar. Kirchhoff pressure distribution. (a) Q1/P0 bilinear displacement/constant pressure quadrilateral element, (b) P1 irreductible displacements triangular element, (c) P1/P1 standard mixed linear displacements/linear pressure triangular element, (d) P1/P1 OSGS stabilized mixed linear displacements/linear pressure triangular element.

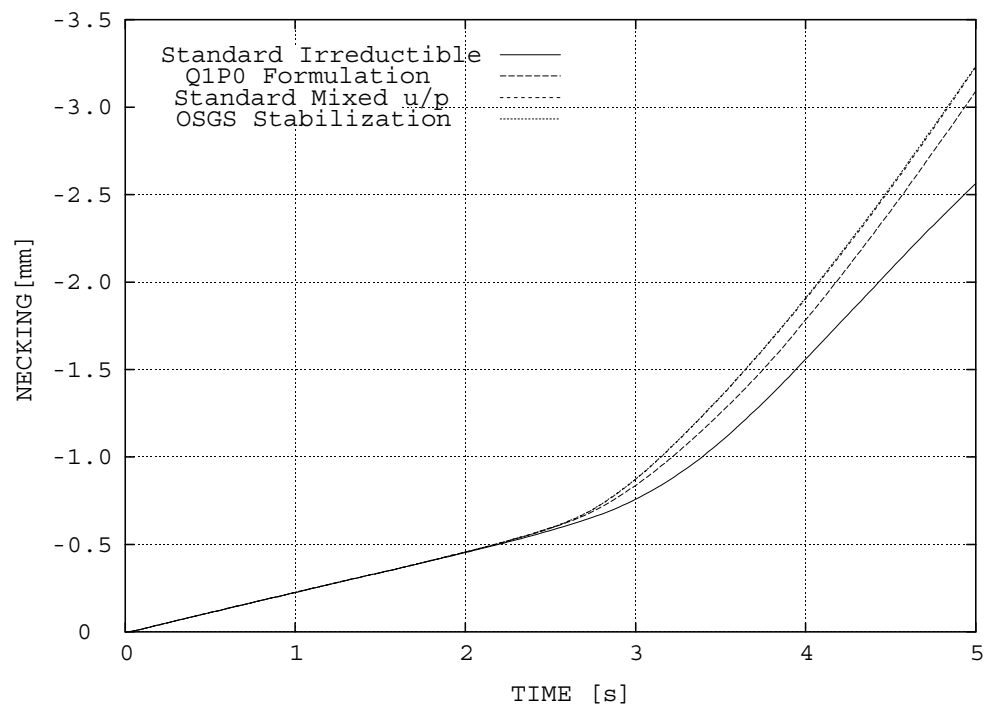

Fig. 5. Plane strain tensile test of a rectangular bar. Necking displacement vs time. 


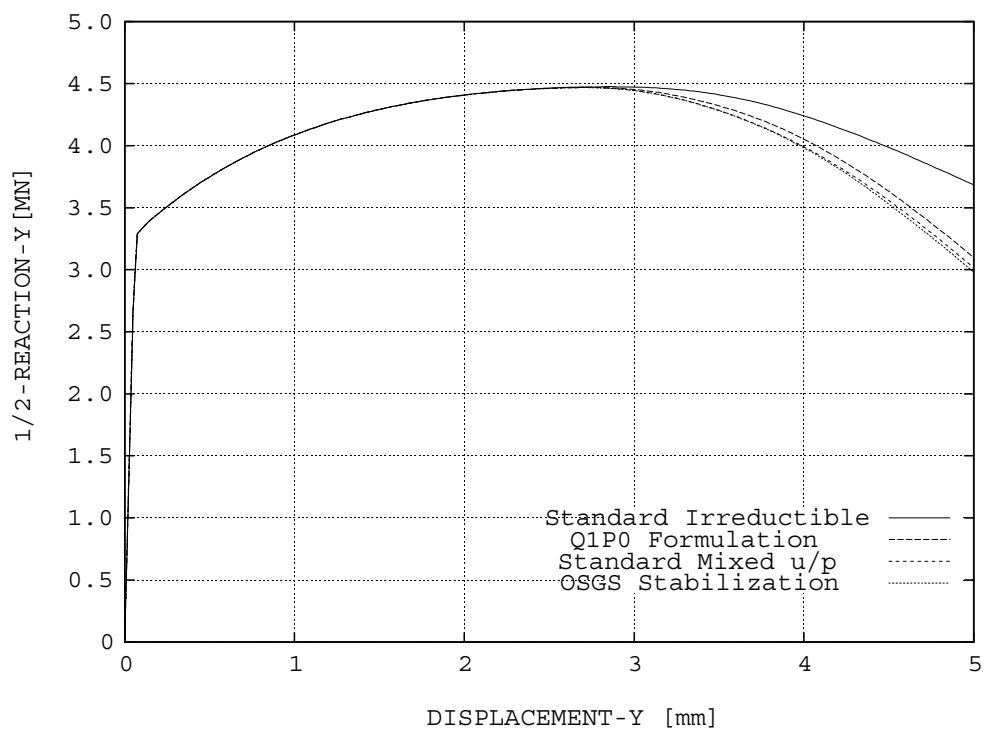

Fig. 6. Plane strain tensile test of a rectangular bar. Pulling reaction vs time.
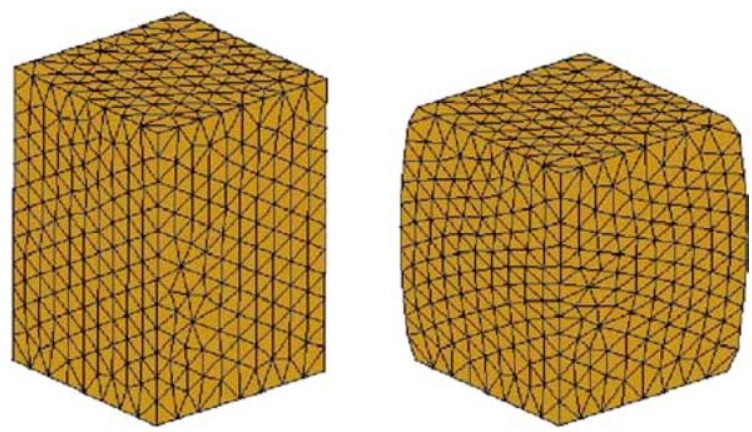

Fig. 7. Upsetting of a 3D block. External view of a quarter part of the initial and deformed geometry discretized using a mesh of tetrahedral elements.

Table 5

Upsetting of a 3D block, material properties

\begin{tabular}{llll}
\hline Young's modulus & $E$ & $1.96 E+05$ & $\mathrm{MPa}$ \\
Poisson's coefficient & $v$ & 0.33 & $\mathrm{MPa}$ \\
Initial flow stress & $\sigma_{0}$ & 150 & $\mathrm{MPa}$ \\
Residual flow stress & $\sigma_{\infty}$ & 180 & $\mathrm{MPa}$ \\
Linear hardening coefficient & $H$ & 0 & 0.7 \\
Saturation hardening exponent & $\delta$ & & \\
\hline
\end{tabular}

Fig. 8 shows the Kirchhoff pressure distribution obtained using: (a) Q1P0 mixed trilinear displacements/ constant pressure hexahedral element; (b) P1 standard linear displacements tetrahedral element; (c) P1/P1 mixed linear displacements/linear pressure tetrahedral element; and (d) P1/P1 OSGS stabilized mixed linear displacement/linear pressure tetrahedral element. As it is clearly shown, not only the standard P1 tetrahe- 

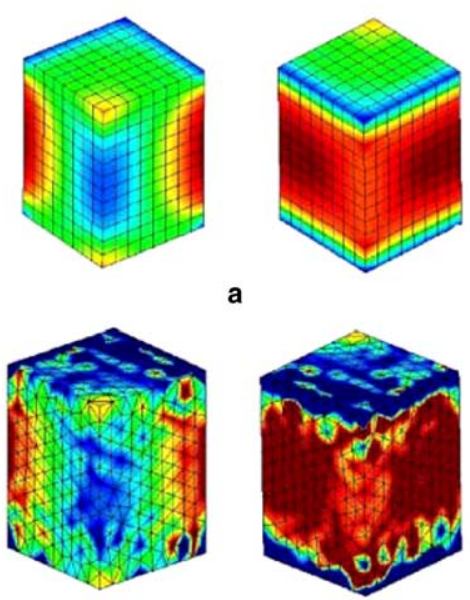

c

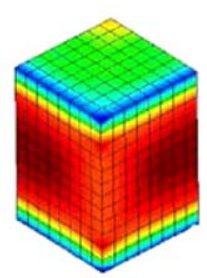

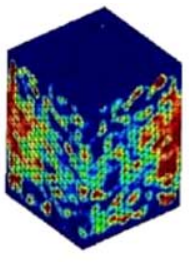

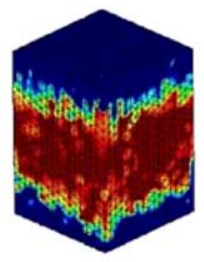

b
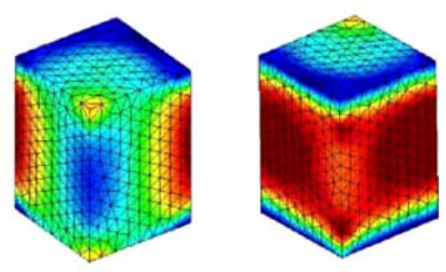

d

Fig. 8. Upsetting of a 3D block. Kirchhoff pressure distribution. Inner and outer views of a quarter part: (a) Q1/P0 mixed bilinear displacements/constant pressure hexahedral element; (b) P1 standard linear displacements tetrahedral element; (c) P1/P1 mixed linear displacements/linear pressure tetrahedral element; (d) P1/P1 OSGS stabilized mixed linear displacements/linear pressure tetrahedral element.

dral element, but also the standard mixed P1/P1 tetrahedral element, give an unstable response with uncontrolled oscillations of the Kirchhoff pressure distribution, while the P1/P1 OSGS stabilized tetrahedral element proposed, as well as the Q1P0 hexahedral element, provide stable pressure distributions.

\subsection{Necking of a circular bar}

This experimentally well-documented example is concerned with the necking of a circular bar, with a radius of $6.413 \mathrm{~mm}$ and length $53.334 \mathrm{~mm}$, subjected to uniaxial tension [36-39]. Loading is imposed using displacement control. A maximum vertical displacement of $7 \mathrm{~mm}$ is applied at the top and bottom edges of the bar. A small geometric imperfection ( $0.982 \%$ of the radius) is introduced at the center of the bar and linearly extended to the top in order to trigger the necking. The material model is assumed to be elastoplastic at finite deformations. Elastic response is given by the stored energy (1). Plastic response is modeled by a J2-flow theory with linear and saturation isotropic hardening. Material properties are summarized in Table 6 [39].

Fig. 9 shows a detail of the necking on the deformed meshes at the final stage of the deformation, using Q1/P0 fine (13571 nodes and 12000 elements) and coarse (1281 nodes and 960 elements) hexahedral meshes and a P1/P1 OSGS (7015 nodes and 35158 elements) tetrahedral mesh. To properly capture the necking a

Table 6

Necking of a circular bar, material properties

\begin{tabular}{|c|c|c|c|}
\hline Shear modulus & $\mu$ & 80.1938 & $\mathrm{GPa}$ \\
\hline Bulk modulus & $\kappa$ & 164.206 & $\mathrm{GPa}$ \\
\hline Initial flow stress & $\sigma_{0}$ & 0.45 & GPa \\
\hline Residual flow stress & $\sigma_{\infty}$ & 0.715 & $\mathrm{GPa}$ \\
\hline Linear hardening coefficient & $H$ & 0.12924 & $\mathrm{GPa}$ \\
\hline Saturation hardening exponent & $\delta$ & 16.93 & \\
\hline
\end{tabular}




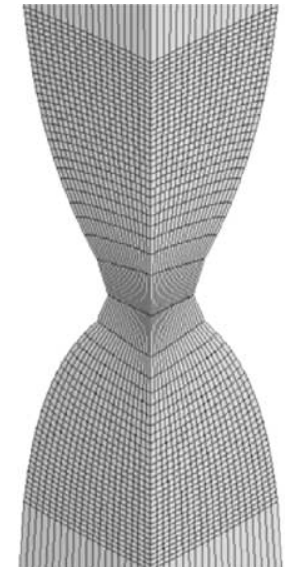

a

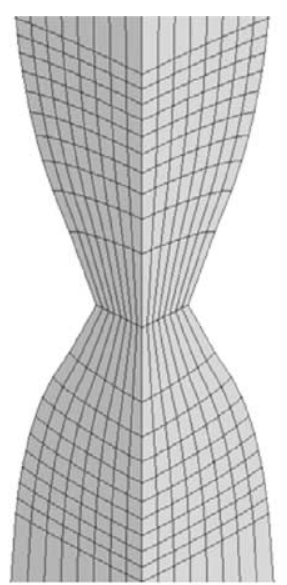

b

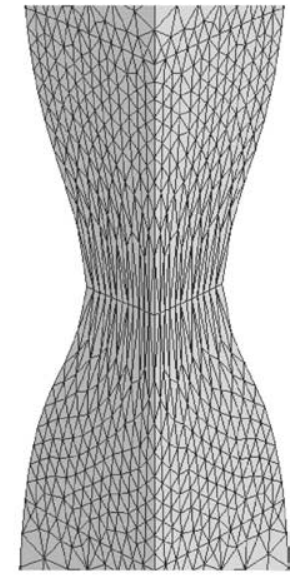

c

Fig. 9. Necking of a circular bar. Details of deformed meshes: (a) Q1/P0 hexahedral element, finer mesh; (b) Q1/P0 hexahedral element and coarse mesh and (c) P1/P1 OSGS tetrahedral element.
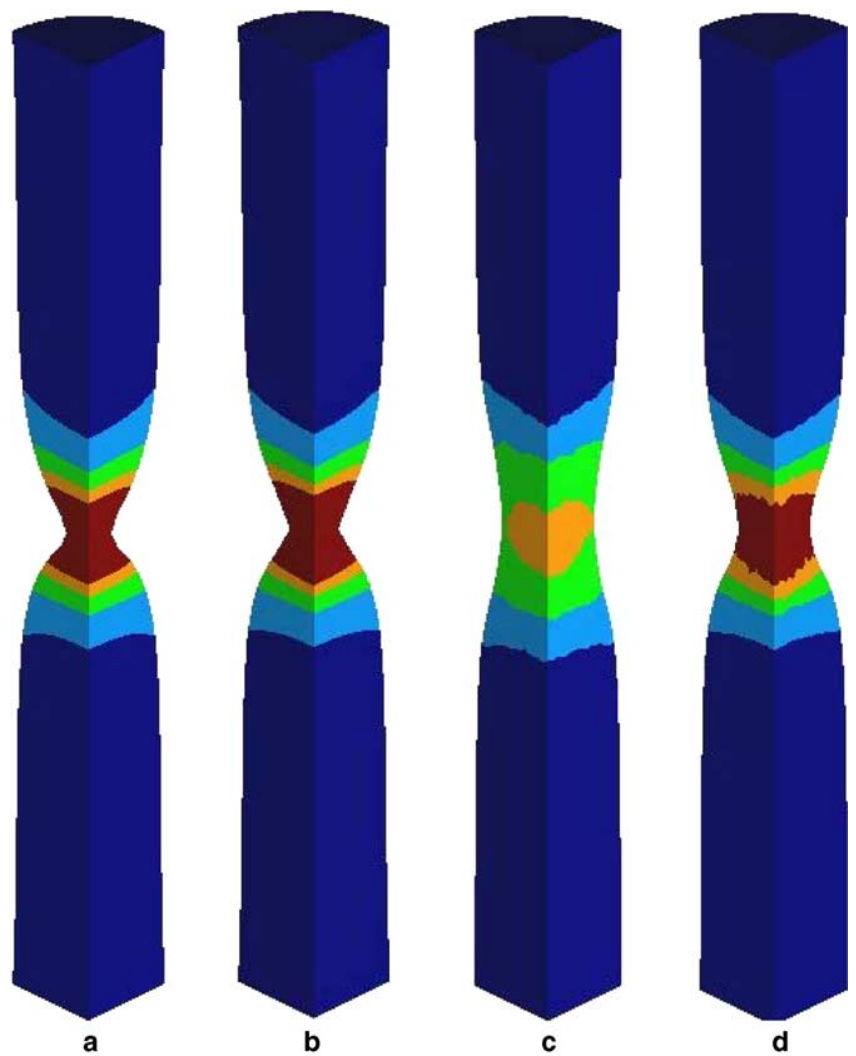

Fig. 10. Necking of a circular bar. Equivalent plastic strain distribution: (a) Q1/P0 hexahedral element, finer mesh; (b) Q1/P0 hexahedral element, coarser mesh; (c) P1 linear displacements tetrahedral element and (d) P1/P1 OSGS tetrahedral element. 
high density of elements is needed in this area. Too coarse meshes at the necking area result in too highly deformed elements and a non-smooth necking pattern [37].

Fig. 10 shows the contours of the equivalent plastic strain at the final stage of the deformation, using Q1/ P0 hexahedral element finer and coarse meshes, P1 tetrahedral elements and P1/P1 OSGS tetrahedral elements. The result provided by the P1/P1 OSGS tetrahedral elements compare very well with the results given by the Q1/P0 hexahedral element. On the other hand, it is clearly shown that the simulation done using $\mathrm{P} 1$ tetrahedral elements is useless.

Fig. 11 shows the contours of the Kirchhoff pressure at the final stage of the deformation, using Q1/P0 fine and coarse hexahedral meshes, P1 tetrahedral elements and P1/P1 OSGS tetrahedral elements. Results provided by the P1/P1 OSGS tetrahedral element compare well with the results given by the Q1/P0 hexahedral element. Results provided by P1 tetrahedral element show lack of stability in the form of uncontrollable oscillations of the Kirchhoff pressure that pollute the solution. Stability is recovered and pressure oscillations are removed using the proposed P1/P1 OSGS mixed stabilized tetrahedral element.

Fig. 12 shows the force (1/4 reaction) vs displacement curves obtained using Q1/P0 coarse hexahedral mesh, P1 tetrahedral elements and P1/P1 OSGS tetrahedral elements. Comparing the curves obtained using the same mesh discretization of P1 and P1/P1 OSGS tetrahedral elements, it can be clearly shown again that the proposed stabilized formulation avoids the locking behaviour typical of the standard irreductible formulation. Load-displacement curves obtained using hexahedral and tetrahedral mesh discretizations are difficult to compare and it is not intended in this figure, to conclude that the Q1/P0 mesh discretization gives
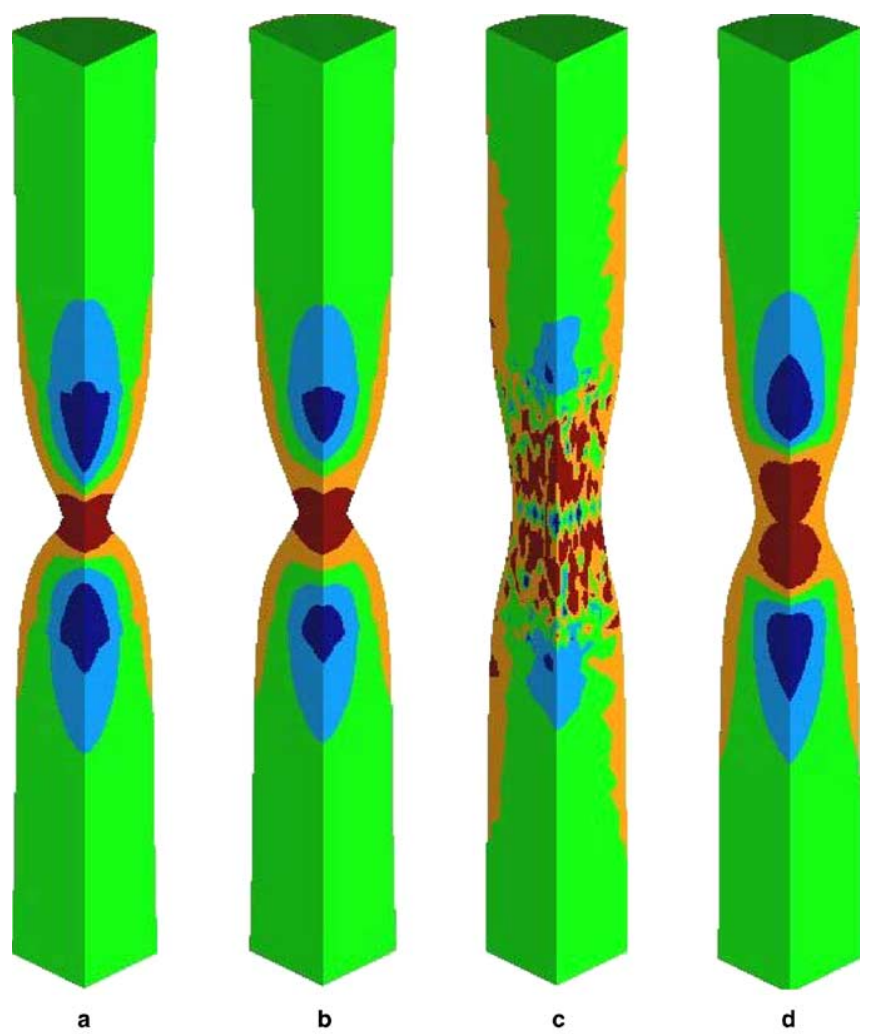

Fig. 11. Necking of a circular bar. Kirchhoff pressure distribution: (a) Q1/P0 hexahedral element, finer mesh; (b) Q1/P0 hexahedral element, coarser mesh; (c) P1 linear displacements tetrahedral element and (d) P1/P1 OSGS tetrahedral element. 


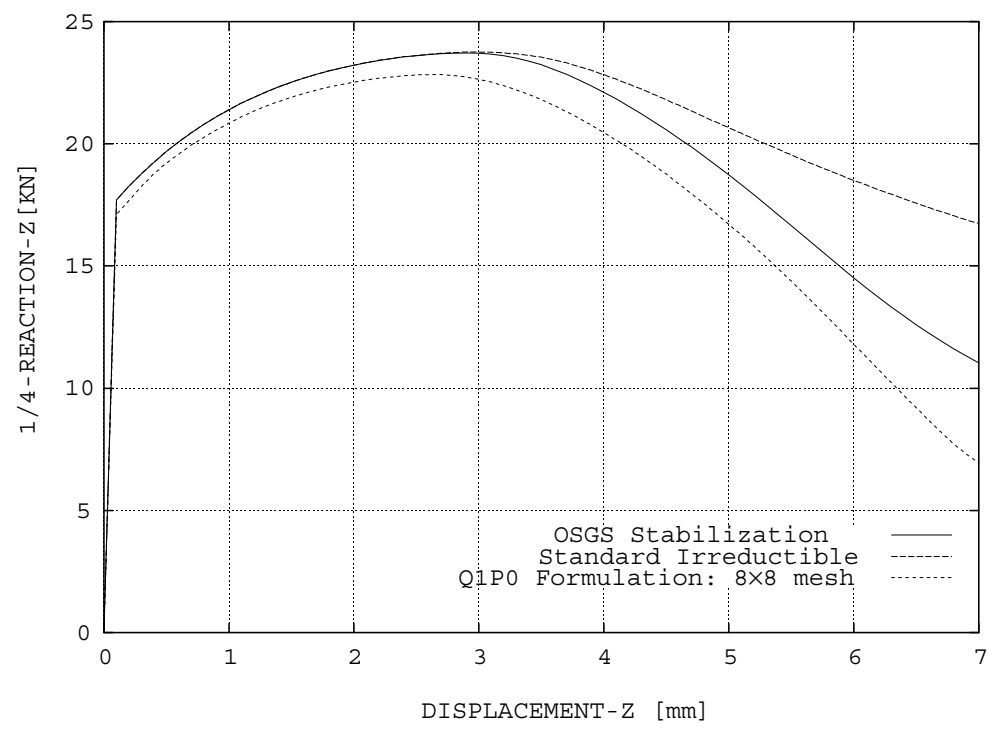

Fig. 12. Necking of a circular bar. Force vs. displacement curves.

a better accuray. Actually different peak and final values of the force in the load-displacement curves are obtained for different Q1/P0 mesh discretizations, as well as for mesh discretizations with the same number of elements but using uniform or non-uniform mesh distributions in the necking area [37].

\section{Concluding remarks}

In this paper a stabilization technique for incompressible J2-flow theory plasticity, within the framework of finite deformation theory, has been presented. The stabilization technique, which falls within the variational multiscale technique, is based on the Orthogonal Subgrid Scale (OSGS) method. Within the paradigmatic framework of the multiscale techniques the Subgrid Scale (SGS) method seeks to approximate the effect of the component of the continuous solution which can not be captured by the finite element mesh used to obtain the discrete finite element solution. The unresolved component is referred as the subgrid scale or subscale. Within the OSGS method we take the orthogonal space to the finite element solution space as the natural space of the subgrid scales. An approximate solution for the subgrid scales is considered and a suitable simple nonlinear expression for the stabilization parameter is proposed. Computational aspects and details of implementation have been shown. Computational simulations show the serious deficiencies of the standard formulations and the huge improvement achieved with the proposed stabilization technique. P1 standard Galerkin displacements element and P1/P1 standard mixed linear displacements/linear pressure element show lack of stability and uncontrolled oscillations of the pressure. The proposed P1/P1 OSGS stabilized element allows to completely remove the pressure oscillations providing pressure stable results within the framework of an elastoplastic J2-flow theory model at finite deformations.

\section{Acknowledgements}

The authors are thankful for the financial support given by European Commission through the Growth project GRD1-2000-25243. Useful discussions with Prof. R. Codina and Prof. E. Oñate are gratefully acknowledged. 


\section{Appendix A. Linearization of the variational momentum balance residual}

Consider the (mixed) variational form of the momentum balance residual equation given by

$$
R_{u}(\mathbf{u}, \pi ; \mathbf{v})=(\pi, \nabla \cdot \mathbf{v})+\left(\mathbf{s}(\mathbf{u}), \nabla^{s} \mathbf{v}\right)-(\mathbf{f}, \mathbf{v})-\left(\overline{\mathbf{t}}^{N}, \mathbf{v}\right)_{\partial \Omega} \quad \forall \mathbf{v} \in \mathscr{V}_{0} .
$$

Using the following key expressions:

$$
\begin{aligned}
& \tau=\mathbf{P F}^{\mathrm{T}} \\
& \nabla \mathbf{v}=\operatorname{Grad}[\mathbf{v}] \mathbf{F}^{-1},
\end{aligned}
$$

where $\mathbf{P}$ is the first Piola-Kirchhoff stress tensor and Grad[·] denotes the material gradient operator, and taking into account that $(\pi, \nabla \cdot \mathbf{v})+\left(\mathbf{s}(\mathbf{u}), \nabla^{s} \mathbf{v}\right)=\left(\tau, \nabla^{s} \mathbf{v}\right)$, the following result holds:

$$
\left(\tau, \nabla^{s} \mathbf{v}\right)=\left(\mathbf{P F}^{\mathrm{T}}, \operatorname{Grad}[\mathbf{v}] \mathbf{F}^{-1}\right)=(\mathbf{P}, \operatorname{Grad}[\mathbf{v}]) .
$$

Using (53), the variational form (50) can be written as

$$
R_{u}(\mathbf{u}, \pi ; \mathbf{v}):=(\mathbf{P}(\mathbf{u}, \pi), \operatorname{GRAD}[\mathbf{v}])-(\mathbf{f}, \mathbf{v})-\left(\overline{\mathbf{t}}^{N}, \mathbf{v}\right)_{\partial \Omega} \quad \forall \mathbf{v} \in \mathscr{V}_{0} .
$$

Then the linearization of (50), using the equivalent expression (54), can be written as

$$
D R_{u}(\mathbf{u}, \pi ; \mathbf{v}) \cdot(\Delta \mathbf{u}, \Delta \pi)=(D \mathbf{P}(\mathbf{u}, \pi) \cdot(\Delta \mathbf{u}, \Delta \pi), \operatorname{Grad}[\mathbf{v}]),
$$

where $D R_{u}(\mathbf{u}, \pi ; \mathbf{v}) \cdot(\Delta \mathbf{u}, \Delta \pi)$ and $D \mathbf{P}(\mathbf{u}, \pi) \cdot(\Delta \mathbf{u}, \Delta \pi)$ denote the directional derivatives of the residual and first Piola-Kirchhoff stress tensor, respectively, along the directions $\Delta \mathbf{u}$ and $\Delta \pi$. Let us compute now the linearization of the first Piola-Kirchhoff stress tensor.

Linearization of the first Piola-Kirchhoff stress tensor. The first Piola-Kirchhoff stress tensor $\mathbf{P}$ can be expressed in terms of the second Piola-Kirchhoff stress tensor $\mathbf{S}$ and the deformation gradient $\mathbf{F}$ as

$$
\mathbf{P}=\mathbf{F S} \text {. }
$$

Then the linearization of the first Piola-Kirchhoff stress tensor yields

$$
D \mathbf{P} \cdot(\Delta \mathbf{u}, \Delta \pi)=D \mathbf{F} \cdot \Delta \mathbf{u} \mathbf{S}+\mathbf{F} D \mathbf{S} \cdot(\Delta \mathbf{u}, \Delta \pi),
$$

where the variation of the deformation gradient $D \mathbf{F} \cdot \Delta \mathbf{u}$ and the variation of the second Piola-Kirchhoff stress tensor $D \mathbf{S} \cdot(\Delta \mathbf{u}, \Delta \pi)$ take the form

$$
\begin{aligned}
& D \mathbf{F} \cdot \Delta \mathbf{u}=\nabla \Delta \mathbf{u F}, \\
& D \mathbf{S} \cdot(\Delta \mathbf{u}, \Delta \pi)=\mathbf{F}^{-1} \mathscr{L}_{\Delta \mathbf{u}} \tau \mathbf{F}^{-\mathrm{T}},
\end{aligned}
$$

where $\mathscr{L}_{\Delta \mathbf{u}} \tau:=\Delta \tau-\nabla(\Delta \mathbf{u}) \tau-\tau(\nabla \Delta \mathbf{u})^{\mathrm{T}}$ denotes the Lie derivative of the Kirchhoff stress tensor along the flow induced by $\Delta \mathbf{u}$. Introducing the split of the Kirchhoff stress tensor into its spherical (in terms of the Kirchhoff pressure $\pi$ ) and deviatoric parts $\tau=\pi \mathbf{1}+\mathbf{s}$, the Lie derivative yields

$$
\mathscr{L}_{\Delta \mathbf{u}} \tau=\mathscr{L}_{\Delta \mathbf{u}}(\pi \mathbf{1})+\mathscr{L}_{\Delta \mathbf{u}} \mathbf{s},
$$

where

$$
\begin{aligned}
& \mathscr{L}_{\Delta \mathbf{u}}(\pi \mathbf{1})=\Delta \pi \mathbf{1}-2 \pi \nabla^{s} \Delta \mathbf{u}, \\
& \mathscr{L}_{\Delta \mathbf{u}} \mathbf{s}=\Delta \mathbf{s}-\nabla(\Delta \mathbf{u}) \mathbf{s}-\mathbf{s}(\nabla \Delta \mathbf{u})^{\mathrm{T}}=\mathbf{c}^{\mathrm{dev}}: \nabla^{s}(\Delta \mathbf{u}),
\end{aligned}
$$

where the incremental constitutive equation for the deviatoric part of the Kirchhoff stress tensor $\mathscr{L}_{\Delta \mathbf{u}} \mathbf{s}=\mathbf{c}^{\mathrm{dev}}: \nabla^{s}(\Delta \mathbf{u})$ has been introduced. 
Substituting (57) and (58) into (56) yields

$$
D \mathbf{P} \cdot(\Delta \mathbf{u}, \Delta \pi)=\nabla \Delta \mathbf{u} \mathbf{F S F}^{\mathrm{T}} \mathbf{F}^{-\mathrm{T}}+\mathbf{F F}^{-1} \mathscr{L}_{\Delta \mathbf{u}} \tau \mathbf{F}^{-\mathrm{T}}=\left[\nabla \Delta \mathbf{u} \tau+\mathscr{L}_{\Delta \mathbf{u}} \tau\right] \mathbf{F}^{-\mathrm{T}} .
$$

Linearization of the variational form of the residual. Substituting the linearization of the first Piola-Kirchhoff stress tensor given by (59) into (55) and using (52), yields

$$
(D \mathbf{P} \cdot(\Delta \mathbf{u}, \Delta \pi), \operatorname{GRAD}[\mathbf{v}])=\left(\left[\nabla \Delta \mathbf{u} \tau+\mathscr{L}_{\Delta \mathbf{u}} \tau\right] \mathbf{F}^{-\mathrm{T}}, \operatorname{Grad}[\mathbf{v}]\right)=(\nabla \Delta \mathbf{u} \tau, \nabla \mathbf{v})+\left(\mathscr{L}_{\Delta \mathbf{u}} \tau, \nabla^{s} \mathbf{v}\right) .
$$

Introducing the split of the Kirchhoff stress tensor $\tau=\pi \mathbf{1}+\mathbf{s}$ and using (57) and (58) yields

$$
(D \mathbf{P} \cdot(\Delta \mathbf{u}, \Delta \pi), \operatorname{Grad}[\mathbf{v}])=(\nabla \Delta \mathbf{u}(\pi \mathbf{1}+\mathbf{s}), \nabla \mathbf{v})+(\Delta \pi, \nabla \cdot \mathbf{v})-\left(2 \pi \nabla^{s} \Delta \mathbf{u}, \nabla^{s} \mathbf{v}\right)+\left(\mathbf{c}^{\operatorname{dev}}: \nabla^{s} \Delta \mathbf{u}, \nabla^{s} \mathbf{v}\right) .
$$

\section{References}

[1] D.N. Arnold, F. Brezzi, M. Fortin, A stable finite element for the Stokes equations, Calcolo 21 (1984) 337-344.

[2] C. Baiocchi, F. Brezzi, L.P. Franca, Virtual bubbles and Galerkin/least-square type methods, Comput. Methods Appl. Mech. Engrg. 105 (1993) 125-141.

[3] F. Brezzi, M. Fortin, Mixed and Hybrid Finite Element Methods, Spinger, New York, 1991.

[4] F. Brezzi, M.O. Bristeau, L.P. Franca, M. Mallet, G. Rogé, A relationship between stabilized finite element methods and the Galerkin method with bubble functions, Comput. Methods Appl. Mech. Engrg. 96 (1992) 117-129.

[5] M. Cervera, C. Agelet de Saracibar, M. Chiumenti, COMET-a COupled MEchanical and Thermal analysis code. Data input manual, Version 5.0, Technical Report IT-308, CIMNE. Available from <http://www.cimne.upc.es>, 2002.

[6] M. Cervera, M. Chiumenti, Q. Valverde, C. Agelet de Saracibar, Mixed linear/linear simplicial elements for incompressible elasticity and plasticity, Comput. Methods Appl. Mech. Engrg. 192 (2003) 5249-5263.

[7] M. Cervera, M. Chiumenti, C. Agelet de Saracibar, Softening localization and stabilization: capture of discontinuous solutions in J2 plasticity, Int. J. Numer. Anal. Methods Geomech. 28 (2004) 373-393.

[8] M. Cervera, M. Chiumenti, C. Agelet de Saracibar, Shear band localization via local J2 continuum damage mechanics, Comput. Methods Appl. Mech. Engrg. 193 (2004) 849-880.

[9] M. Cervera, M. Chiumenti, C. Agelet de Saracibar, Shear band localization via local J2 continuum damage mechanics, Monograph M78, CIMNE, 2003.

[10] D. Christ, M. Cervera, M. Chiumenti, C. Agelet de Saracibar, A mixed finite element formulation for incompressibility using linear displacement and pressure interpolations, Monograph 77, CIMNE, 2003.

[11] M. Chiumenti, Q. Valverde, C. Agelet de Saracibar, M. Cervera, A stabilized formulation for incompressible elasticity using linear displacement and pressure interpolations, Comput. Methods Appl. Mech. Engrg. 191 (2002) 5253-5264.

[12] M. Chiumenti, Q. Valverde, C. Agelet de Saracibar, M. Cervera, A stabilized formulation for incompressible plasticity using linear triangles and tetrahedra, Int. J. Plast. 20 (2004) 1487-1504.

[13] R. Codina, Stabilization of incompressibility and convection through orthogonal sub-scales in finite element methods, Comput. Methods Appl. Mech. Engrg. 190 (2000) 1579-1599.

[14] R. Codina, Stabilized finite element approximation of transient incompressible flows using orthogonal subscales, Comput. Methods Appl. Mech. Engrg. 191 (2002) 4295-4321.

[15] R. Codina, J. Blasco, Stabilized finite element method for transient Navier-Stokes equations based on pressure gradient projection, Comput. Methods Appl. Mech. Engrg. 182 (2000) 287-300.

[16] K. Garikipati, T.J.R. Hughes, A study of strain localization in a multiple scale framework-the one dimensional problem, Comput. Methods Appl. Mech. Engrg. 159 (1998) 193-222.

[17] K. Garikipati, T.J.R. Hughes, A variational multiscale approach to strain localization-formulation for multidimensional problems, Comput. Methods Appl. Mech. Engrg. 188 (2000) 39-60.

[18] GiD: The personal pre and postprocessor. Available from <http://www.cimne.upc.es>, 2002.

[19] T.J.R. Hughes, The Finite Element Method: Linear Static and Dynamic Finite Element Analysis, Prentice-Hall Inc., Englewood Cliffs, NJ, 1987.

[20] T.J.R. Hughes, Multiscale phenomena: Green's function, Dirichlet-to-Neumann formulation, subgrid scale models, bubbles and the origins of stabilized formulations, Comput. Methods Appl. Mech. Engrg. 127 (1995) 387-401.

[21] T.J.R. Hughes, L.P. Franca, M. Balestra, A finite element formulation for computational fluid dynamics: V. Circumventing the Babuška-Brezzi condition: a stable Petrov-Galerkin formulation of the Stokes problem accomodating equal-order interpolations, Comput. Methods Appl. Mech. Engrg. 59 (1986) 85-99. 
[22] T.J.R. Hughes, L.P. Franca, G.M. Hulbert, A new finite element formulation for computational fluid dynamics: VIII. The Galerkin/least-square method for advective-diffusive equations, Comput. Methods Appl. Mech. Engrg. 73 (1989) $173-189$.

[23] T.J.R. Hughes, G.R. Feijóo, L. Mazzei, J.-B. Quincy, The variational multiscale method-a paradigm for computational mechanics, Comput. Methods Appl. Mech. Engrg. 166 (1998) 3-24.

[24] O. Klaas, A. Maniatty, M.S. Shephard, A stabilized mixed finite element method for finite elasticity. Formulation for linear displacement and pressure interpolation, Comput. Methods Appl. Mech. Engrg. 180 (1999) 65-79.

[25] A. Maniatty, Y. Liu, Stabilized finite element method for viscoplastic flow: formulation with state variable evolution, Int. J. Numer. Methods Engrg. 56 (2003) 185-209.

[26] A. Maniatty, Y. Liu, O. Klaas, M.S. Shephard, Stabilized finite element method for viscoplastic flow: formulation and a simple progressive solution strategy, Comput. Methods Appl. Mech. Engrg. 190 (2001) 4609-4625.

[27] A. Maniatty, Y. Liu, O. Klaas, M.S. Shephard, Higher order stabilized finite element method for hyperelastic finite deformation, Comput. Methods Appl. Mech. Engrg. 191 (2002) 1491-1503.

[28] C. Miehe, Aspects of the formulation and finite element implementation of large strain isotropic elasticity, Int. J. Numer. Methods Engrg. 37 (1994) 1981-2004.

[29] E. Oñate, J. Rojek, R.L. Taylor, O.C. Zienkiewicz, Linear triangles and tetrahedra for incompressible problem using a finite calculus formulation, in: Proceedings of European Conference on Computational Mechanics, ECCM 2001, 2001.

[30] E. Oñate, J. Rojek, R.L. Taylor, O.C. Zienkiewicz, Finite calculus formulation for incompressible solids using linear triangles and tetrahedra, Int. J. Numer. Methods Engrg. 59 (2004) 1473-1500.

[31] B. Ramesh, A. Maniatty, Stabilized finite element formulation for elasto-plastic finite deformations, Comput. Methods Appl. Mech. Engrg. 194 (2005) 775-800.

[32] B.D. Reddy, J.C. Simo, Stability and convergence of a class of enhanced assumed strain methods, SIAM J. Numer. Anal. 32 (1995) 1705-1728.

[33] S. Reese, P. Wriggers, A stabilization technique to avoid hourglassing in finite elasticity, Int. J. Numer. Methods Engrg. 48 (2000) 79-109.

[34] S. Reese, M. Küssner, B.D. Reddy, A new stabilization technique for finite elements in nonlinear elasticity, Int. J. Numer. Methods Engrg. 44 (1999) 1617-1652.

[35] J.C. Simo, A framework for finite strain elastoplasticity based on maximum plastic dissipation and the multiplicative decomposition: Part I. Continuum formulation, Comput. Methods Appl. Mech. Engrg. 66 (1988) 199-219.

[36] J.C. Simo, A framework for finite strain elastoplasticity based on maximum plastic dissipation and the multiplicative decomposition: Part II. Computational aspects, Comput. Methods Appl. Mech. Engrg. 68 (1988) 1-31.

[37] J.C. Simo, F. Armero, Geometrically non-linear enhanced strain mixed methods and the method of incompatible modes, Int. J. Numer. Methods Engrg. 33 (1992) 1413-1449.

[38] J.C. Simo, F. Armero, R.L. Taylor, Improved versions of assumed enhanced strain tri-linear elements for 3D finite deformations, Comput. Methods Appl. Mech. Engrg. 110 (1993) 359-386.

[39] J.C. Simo, T.J.R. Hughes, Computational Inelasticity, Springer, Berlin, 1998.

[40] J.C. Simo, C. Miehe, Associative coupled thermoplasticity at finite strains: formulation, numerical analysis and implementation, Comput. Methods Appl. Mech. Engrg. 98 (1992) 41-104.

[41] J.C. Simo, M.S. Rifai, A class of mixed assumed strain methods and the method of incompatible modes, Int. J. Numer. Methods Engrg. 29 (1990) 1595-1638.

[42] J.C. Simo, R.L. Taylor, K.S. Pister, Variational and projection methods for the volume constraint in finite deformation elastoplasticity, Comput. Methods Appl. Mech. Engrg. 51 (1985) 177-208.

[43] R.L. Taylor, A mixed formulation for triangular and tetrahedral elements, in: Métodos Numéricos en Ingeniería Conference Proceedings, SEMNI 1999, Barcelona, Spain, 1999.

[44] Q. Valverde, C. Agelet de Saracibar, M. Cervera, M. Chiumenti, Elementos estabilizados de bajo orden en mecánica de sólidos, Monografía M 69, CIMNE, 2002.

[45] O.C. Zienkiewicz, R.L. Taylor, Fifth ed.The Finite Element Method, Vol. 1-3, Butterworth-Heinemann, London, 2000.

[46] O.C. Zienkiewicz, J. Rojek, R.L. Taylor, M. Pastor, Triangles and tetrahedra in explicit dynamic codes for solids, Int. J. Numer. Methods Engrg. 43 (1998) 565-583. 